\title{
WILEY-VCH
}

DOI: 10.1002/ ((please add manuscript number))

Article type: Full Paper

\section{Nanoscale thermal transport in 2D nanostructures from cryogenic to room temperatures}

Charalambos Evangeli*, Jean Spiece, Hatef Sadeghi*, Sara Sangtarash, Aday J. MolinaMendoza, Marta Mucientes, Thomas Mueller, Colin Lambert and Oleg Kolosov*

Dr. C. Evangeli*, Dr. J.Spiece, Dr. H. Sadeghi, Dr. S. Sangtarash, M. Mucientes, Prof. C. Lambert, Prof. O. Kolosov

Physics Department, Lancaster University, Lancaster, LA1 4YB, UK

E-mail: o.kolosov@lancaster.ac.uk; charalambos.evangeli@materials.ox.ac.uk;

h.sadeghi@lancaster.ac.uk.

Dr. A. J. Molina-Mendoza, Prof. T. Mueller

Institute of Photonics, Vienna University of Technology, Gusshausstrasse 27-29, Vienna, A1040, Austria.

Keywords: graphene, $\mathrm{MoS}_{2}$, scanning thermal microscopy, thermal conductivity, cryogenic

Nanoscale Scanning Thermal Microscopy (SThM) transport measurements from cryogenic to room temperature on two-dimensional (2D) structures with sub $30 \mathrm{~nm}$ resolution are reported. This novel cryogenic operation of SThM, extending the temperature range of the sample down to $150 \mathrm{~K}$, yields a clear insight into the nanothermal properties of the $2 \mathrm{D}$ nanostructures and supports the model of ballistic transport contribution at the edge of the detached areas of exfoliated graphene which leads to a size-dependent thermal resistance of the detached material. The thermal resistance of graphene on $\mathrm{SiO}_{2}$ is increased by one order of magnitude by the addition of a top layer of $\mathrm{MoS}_{2}$, over the temperature range of $150-300 \mathrm{~K}$, providing pathways for increasing the efficiency of thermoelectric applications using van der Waals $(\mathrm{vdW})$ materials. Density functional theory calculations demonstrate that this increase originates from the phonon transport filtering in the weak vdW coupling between the layers and the vibrational mismatch between $\mathrm{MoS}_{2}$ and graphene layers. 


\section{WILEY-VCH}

\section{Introduction}

The unique thermal and electronic properties of two-dimensional (2D) materials originate from their atomic scale thickness and uniformity and variety of materials involved, are opening new paradigms for successful miniaturization of electronic devices. In particular, 2D materials are potential alternatives for heat management utilizing active layers in such devices. ${ }^{[1]}$ For example, 2D materials with high in-plane thermal conductivity such as graphene, which is also highly electrically conductive, or hexagonal boron nitride (hBN), which is electrically insulating, have been proposed for heat management applications, ${ }^{[2]}$ whereas $\mathrm{MoS}_{2}$ has been used as an active channel on electronic devices due to its comparable bandgap to silicon. ${ }^{[3]} 2 \mathrm{D}$ materials can also be excellent thermal insulators for cross-plane thermal transport, with WSe 2 known to possess one of the lowest thermal conductivity for a continuous solid-state material. ${ }^{[4]}$ Furthermore, vertical ${ }^{[5]}$, lateral ${ }^{[6]}$ or composite ${ }^{[7]}$ heterostructures of $2 \mathrm{D}$ materials are shown to be potential candidates for electronic applications. These unique properties open new possibilities for the development of high-performance thermoelectric and phase change memory structures.

For thermoelectric applications, 2D materials are also highly attractive due to their high Seebeck coefficient values. For example, the Seebeck coefficient of graphene has been reported to be between $10-180 \mu \mathrm{V} / \mathrm{K}^{[8]}$ while that of $\mathrm{MoS}_{2}$ can be orders of magnitude higher $(3 \times$ $\left.10^{5} \mu \mathrm{V} / \mathrm{K}\right) .{ }^{[9]}$ Nevertheless, their implementation in such devices needs clever use of thermal anisotropy of the in-plane $e^{[10]}$ and cross-plane ${ }^{[11]}$ thermal conductivities. The efficiency of a thermoelectric device, which converts the waste heat to energy is determined by the dimensionless figure of merit $Z T=S^{2} \sigma T / k$, where $S$ is the Seebeck coefficient, $\sigma$ is the electrical conductivity and $k=k_{p h}+k_{e l}$ is the thermal conductivity due to electrons (el) and phonons (ph). Therefore, a highly efficient thermoelectric device requires materials with high $S$ and $\sigma$ and low $k$ in the direction of the electron flow. 


\section{WILEY-VCH}

Recently, it has been proposed that stacking of 2D materials to create heterostructures is an efficient way of enhancing the efficiency of thermoelectric devices. ${ }^{[12]}$ For graphene/MoS 2 stacks, high figure of merit values (up to 2.8) are predicted, due to the reduction of $k$ and heat transport through the sharp edges of $\mathrm{MoS}_{2}$ nanoribbons. ${ }^{[13]}$ Additionally, the thermal conductivity of rippled graphene was predicted to notably drop compare to flat one ${ }^{[14]}$ leading to more efficient thermoelectrics. It has also been predicted that reduced thermal conductivity can be achieved by engineering the thermal transport in graphene periodic phononic structures. ${ }^{[15]}$ Furthermore, it has been reported that lateral confinement alone allows to change the Seebeck coefficient in 2D materials paving the way to the new generation of thermoelectrics. ${ }^{[16]}$

To experimentally investigate heat transport in such 2D nanostructures, it is essential to develop techniques with both nanoscale lateral resolution and an ability to sensitively evaluate the nanoscale heat transport over an extended temperature range, which is essential for understanding the fundamental nature of such transport, as well as proving pathways for addressing practical solutions. Whereas currently used techniques such as Raman spectroscopy or lithographically fabricated devices allow for such measurements, their lateral resolution is either limited to the micrometre scale (for Raman) ${ }^{[17]}$ or is restricted to a single point of the device. ${ }^{[18]}$ The only technique that allows interactive mapping of thermal transport across all structure with nanoscale resolution is Scanning Thermal Microscopy (SThM), which combines the superior nanoscale lateral resolution of the Atomic Force Microscope (AFM) and sensitivity to nanoscale heat flow by incorporating a resistive heater and temperature sensor close to the tip of an AFM probe. ${ }^{[19]}$ SThM has been employed in several studies for the thermal characterization of $2 \mathrm{D}$ materials in ambient, vacuum or liquid environment, ${ }^{[20]}$ although all measurements reported up to date have been performed at near-ambient sample temperature. In cryogenic temperatures it has only been employed for the measurements of temperature fields $^{[21]}$. 


\section{WILEY-VCH}

Here we present, for the first time, high vacuum SThM thermal transport measurements on graphene/MoS $2 \mathrm{D}$ heterostructures with sub-30 $\mathrm{nm}$ resolution at temperatures varying from $150-300 \mathrm{~K}$. We demonstrate that the effective thermal conductivity of graphene at the nanoscale can be decreased significantly over all the temperature range to a variable degree up to one order of magnitude, by adding a top layer of $\mathrm{MoS}_{2}$. Furthermore, the increased thermal resolution at cryogenic temperatures enabled direct indication of the contribution of ballistic transport at the edge of the detached " $2 \mathrm{D}$ materials bubble" areas ${ }^{[22]}$ of exfoliated graphene leading to a size-dependent thermal resistance of detached 2D material.

\section{Results and Discussion}

\subsection{Cryo-SThM principles/demonstration:}

We modified a specialized High Vacuum $\mathrm{SThM}^{[20 \mathrm{a}]}$ with the sample holder connected through a copper braid to a liquid nitrogen $\left(\mathrm{LN}_{2}\right)$ Dewar (see Figure 1a) allowing us to change the sample temperature $\left(T_{S}\right)$ between room temperature $(300 \mathrm{~K})$ down to $150 \mathrm{~K}$. We use commercially available (Anasys Instruments, AN-300) doped silicon probes (DSi), which have a geometry similar to the standard micromachined Si AFM probes. The probe temperature can be controlled with an integrated heater at the end of the cantilever, which also acts as a temperature sensor. When the tip is in contact with a sample, the temperature of the heater which is monitored changes depending on the heat transfer between the tip and the sample.

To quantify the SThM response and to present thermal images in units of thermal resistance, we record approach- and retract-curves of the SThM signal at each sample temperature immediately after every image (see Figure 1b). To achieve this, the thermal probe is slowly brought towards the sample resulting in a sharp drop of the SThM signal on tip-sample mechanical contact, as the heater temperature changes in response to the heat flowing into the sample. This sharp drop is followed by a further gradual decrease, as a better degree of contact is established ${ }^{[23]}$ (blue curve, Figure 1b). The linearities of relationships between the 


\section{WILEY-VCH}

temperature gradients and the heat transport, as well as heater electrical resistance and its temperature, allow us to link the SThM signal difference for the probe in- and out-of-contact to the thermal resistance of the tip-sample contact through the following expression ${ }^{[24]}$ (see Supporting Information note 1 for a relevant derivation):

$\frac{V_{n c}-V_{c}}{V_{n c}}=\frac{1}{R_{p}+R_{X}}\left(R_{p}+\frac{T_{M}-T_{S}}{Q_{h}}\right)$,

where $V_{n c}$ is the SThM signal when the probe is out-of-contact and $V_{c}$, the signal in contact with the sample, $Q_{h}$ is the heat generated in the probe heater, $R_{X}$ is the heater-sample thermal contact resistance and $R_{p}$ the probe thermal resistance. Importantly, Equation 1 shows that in contrast with typical ambient SThM measurements, where $T_{M}=T_{S}$, in the cryo-SThM, the temperature difference $T_{M}-T_{S}$ between the microscope and the sample needs to be taken into account to correctly interpret the measurements. A typical example in the inset of Figure 1b, shows the relative signal "jump" $\frac{V_{n c}-V_{c}}{V_{n c}}$ for $\mathrm{SiO}_{2}$ on $\mathrm{Si}$ substrate at room temperature (RT) and low temperature (LT) changes three-fold, thereby requiring use of the relationships reflected by the Equation 1.

As $T_{M}$ and $T_{S}$ are monitored during the experiment and $Q_{h}$ and $R_{p}$ are known through the probe calibration, we can calculate $R_{X}$ from Equation (1). Given that $V_{n c}$ does not depend on the position of the probe, then by replacing $V_{c}$ with equivalent SThM signal that is obtained during tip-sample contact at a close to zero contact force, we can quantify the nanoscale SThM map in terms of $R_{X}$. In order to interpret the heat transfer mechanisms monitored during our experiments, it is helpful to present $R_{X}$ as three different resistances in series: ${ }^{[25]}$

$R_{X}=R_{\text {tip }}+R_{\text {int }}+R_{\text {spr }}$

where $R_{\text {tip }}$ is the constriction resistance of the sharp conical tip, $R_{\text {int }}$ is the interfacial resistance between the tip apex and the sample surface and $R_{s p r}$ is the spreading resistance of the sample. We discuss the contribution of these three components and their temperature dependence below. 


\section{WILEY-VCH}

We note that although we can express our measurements in terms of $R_{X}$, one should be aware that the absolute values always depend on the tip-sample contact radius. Therefore, the ability to compare absolute measurements requires the assumption that the tip-sample contact radius is the same. This assumption is typically valid when the same probe is used on materials with similar elastic moduli, and the surface does not have sharp corrugations. It is in particular valid in the case of 2D materials with vertical corrugations on the order of few nm, and lateral dimensions of ca. $100 \mathrm{~nm}$ as shown elsewhere. ${ }^{[20 \mathrm{a}]}$ With the tip and the sample being at very dissimilar temperatures, a natural question arises - whether the temperature of the sample temperature immediately under the tip-surface contact is significantly different from the bulk $T_{S}$. Essentially, as shown elsewhere, ${ }^{[26]}$ for the typical DSi probe and most solid state materials except low thermal conductivity polymers, the thermal resistance of the narrow nanoscale sharp Si tip is the dominating part of the $R_{X}$ resulting in the sample surface remaining at the cryogenic temperature providing a basis for the cryo-SThM. At the same time, this local heating, which is dependent on the thermal resistance of the sample, requires correction in the measurement that we provide in the next section.

Figure $1 \mathrm{c}$ and $\mathrm{d}$ shows a typical example of a high-resolution topography and $R_{X}$ cryoSThM images of a few atomic layers exfoliated graphene on $280 \mathrm{~nm} \mathrm{SiO} / \mathrm{Si}$ substrate. Both images were acquired simultaneously. Exfoliated graphene forms detached areas in the shape of ripples and bubbles ${ }^{[27]}$ as can be seen in the topography image. It is interesting that the $R_{X}$ of graphene bubbles can be higher or lower than the one of attached areas, depending on their surface area (see Supporting Information note 9 for further details). Moreover, a ring of $R_{X}$ appears around the perimeter of each bubble, that is likely related to ballistic contribution to the heat transport in the suspended graphene areas as previously observed in suspended graphene systems. $^{[20 \mathrm{a}, 28]}$ At the same time, $R_{X}$ of a center of a "bubble" area has two components connected in series that have opposite dependences on the "bubble" size. The in-plane sheet 


\section{WILEY-VCH}

resistance that will increase logarithmically with the "bubble" effective diameter ${ }^{[29]}$ and the peripheral resistance inversely proportional to the perimeter of the "bubble" and therefore decreasing with the increase of the bubble size. With the stronger dependence of the inverse power law of peripheral resistance dominating the behaviour, the central area of sufficiently large bubbles show lower thermal resistance as seen in the Figure 1d. At RT, the above findings are barely visible. The difference in resolution has two possible origins: the relative change of graphene thermal transport between attached and detached areas and increase of the phonon mean free path $(\lambda)$ at LT, or an increase of the thermal resistance of the DSi tip, which causes the graphene's thermal conductivity to lie in the sensitive probe regime. ${ }^{[26]}$

\subsection{Graphene/MoS $\mathrm{M}_{2}$ heterostructure:}

To investigate thermal transport at interfaces and in $2 \mathrm{D}$ materials, a single layer graphene/ $\mathrm{MoS}_{2}$ heterostructure on a $\mathrm{SiO}_{2} / \mathrm{Si}$ substrate was imaged using $\mathrm{SThM}$ at various sample temperatures ranging from 150 to $300 \mathrm{~K}$. We have chosen graphene/MoS 2 heterostructure due to its promising predicted thermoelectric properties and low $k \mathrm{SiO}_{2}$ on $\mathrm{Si}$ substrate to avoid ballistic heat transport phenomena related to the substrate. An example of a $R_{X}$ image of the heterostructure at $T_{S}=220 \mathrm{~K}$ is shown in Figure 2a. The sample has areas of bare $\mathrm{SiO}_{2} / \mathrm{Si}$ (SOS), graphene on $\mathrm{SiO}_{2}(\mathrm{GS}), \mathrm{MoS}_{2}$ on $\mathrm{SiO}_{2}$ (MS) and $\mathrm{MoS}_{2}$ on top of GS (MGS). The contrast in the $R_{X}$ image demonstrates the following trend from low to high thermal resistance areas: $R_{X(\mathrm{GS})}<R_{X(S O S)}<R_{X(M G S)}<R_{X(M S)}$. Interestingly, $\mathrm{MoS}_{2}$ increases the thermal resistance of the underlying graphene.

Before analysing the temperature response of the various areas, an interesting result can already be demonstrated. As expected, the high heat conductance of graphene lowers the $R_{X}$ compared to the SOS substrate. Interestingly, $\mathrm{MoS}_{2}$ whose thermal conductivity on $\mathrm{SiO}_{2}$ has been reported to be around $55 \mathrm{Wm}^{-1} \mathrm{~K}^{-1},{ }^{[11 \mathrm{a}, 17]}$ increases the $R_{X}$ of $\mathrm{SiO}_{2}$. One would expect from the $\mathrm{MoS}_{2}$ to lower the $R_{X}$ compare to $\mathrm{SiO}_{2}$ as in the case of graphene. This is because in 


\section{WILEY-VCH}

addition to the layer thermal conductivity, the interfacial thermal resistance plays a role in the thermal transport. ${ }^{[20 \mathrm{~b}]}$ Looking at values reported in the literature for the interfacial resistance between $\mathrm{MoS}_{2}$ or graphene and $\mathrm{SiO}_{2}$, we understand the crucial impact of interfaces on nanoscale thermal transport. The interface $\mathrm{MoS}_{2} / \mathrm{SiO}_{2}$ has values reported between $4 \times 10^{-8}$ to $2.27 \times 10^{-6} \mathrm{~m}^{2} \mathrm{KW}^{-1}[11 \mathrm{a}, 30]$ orders of magnitude higher than graphene/ $\mathrm{SiO}_{2}$ interface resistance with values from $5.6 \times 10^{-9}$ to $2 \times 10^{-8} \mathrm{~m}^{2} \mathrm{KW}^{-1}[18 \mathrm{a}, 31]$. In the following, we develop a model involving both thermal conductivity and interfacial thermal resistance, therefore including both in-plane and cross-plane transport to provide a more precise picture.

The average $R_{X}$ of the different areas was extracted from the corresponding images and are plotted in Figure 2d. All areas including SOS have a similar decreasing trend with increasing temperature. Also the absolute differences of $R_{X(G M S)}$ and $R_{X(M S)}$ with $R_{X(\mathrm{GS})}$ increase with decreasing temperature. In other words, areas with $\mathrm{MoS}_{2}$ increase their $R_{X}$ at lower temperature at a higher rate than areas without $\mathrm{MoS}_{2}$. Therefore, the thermal contrast between the two areas increases, while lowering the temperature. An anomalous point for $R_{X}$ is observed at $T_{S}=$ $190 \mathrm{~K}$ for all areas, which we believe originates from the heat transit thermal phenomena in the silicon tip (see Supporting Information note 2 and 6 for thermal transport in the DSi tip).

Let us first comment on the $R_{X}$ measured on the $\mathrm{SOS}$. The $\mathrm{SiO}_{2}$ thickness grown on $\mathrm{Si}$ wafer is larger than the tip diameter, which we estimated to be at maximum $60 \mathrm{~nm}$ (see Supporting Information note 5). Therefore, the contribution of the underlying Si in the measured $R_{X}$ will be marginal. According to our calculations, the measured $R_{X}$ will be approximately $95 \%$ of the bulk $\mathrm{SiO}_{2}$ (see Supporting Information note 3). As the $\mathrm{SiO}_{2}$ thermal conductivity $k$ is relatively low ${ }^{[32]}$ and its $\lambda$ is small ${ }^{[33]}$ compared to the size of the contact, we can model the system by assuming bulk diffusive heat transfer within the oxide. Taking into account all the above, $\mathrm{SiO}_{2}$ provides a reliable test-bed and can serve as a reliable reference sample for the cryo-SThM measurements. 


\section{WILEY-VCH}

The spreading resistance $R_{s p r}$ in a bulk system is inversely proportional to $k\left(R_{s p r}=\right.$ $\left.1 / 2 k d_{0}\right)$. We can thus plot according to Eq.2, $R_{X S i O 2}(T)=R_{\text {tip }}+R_{\text {int }}+1 / 2 k_{S i O 2}(T) d_{0}$ using temperature dependent thermal conductivity values ${ }^{[32]}$ and $R_{\text {tip }}+R_{\text {int }}$ as a fitting parameter. Comparison between experimental data and this model is presented in Figure $2 \mathrm{c}$ and shows that the thermal resistance variation with temperature measured in the experiment is well reproduced. This highlights and demonstrates the ability of cryo-SThM to measure quantitatively meaningful quantities from RT to LT.

From the above model, $R_{\text {tip }}+R_{\text {int }}$ increases from 1.1 to $1.5 \times 10^{7} \mathrm{KW}^{-1}$ with decreasing temperature (see Supporting Information, Figure SI2). Such values are higher than simple models for conical tip, giving a temperature dependent $R_{t i p}$ varying $0.7-1 \times$ $10^{7} \mathrm{KW}^{-1}$ and $\mathrm{RT}$ literature values of $\mathrm{Si} / \mathrm{SiO}_{2} R_{\text {int }} \cdot{ }^{[34]}$ This difference is not surprising, since effects of multi-asperity tip-surface contact, polymer residues due to the exfoliation process and native $\mathrm{SiO}_{2}$ on the tip would significantly increase $R_{\text {tip }}+R_{\text {int }}$. We performed similar measurements on $\mathrm{Si}$ which, in contrast to $\mathrm{SiO}_{2}$, has high $k$ and long $\lambda$, giving contradictory results in comparison to measurements on bulk samples reported in the literature. ${ }^{[35]}$ We believe that this behaviour originates from the ballistic heat transport contribution to the different components forming $R_{X}$ (see Supporting Information note 6). This also proves that the DSi probe is more sensitive to the sample thermal properties of low $k$ materials, while for high $k$ materials the $R_{\text {int }}$ and geometrical characteristics of the tip-sample contact play an increased role, especially at lower temperatures.

As mentioned above, here we provide approximate corrections for the local heating of the sample due to the excess heat of the tip, which is transferred from the probe, through the 2D structures to the $\mathrm{SiO}_{2}$. The relative SThM signal output "jump" on the contact with the sample is expected to be the same regardless of the probe excess temperature. Although, if the sample is locally heated by the tip, the thermal transport properties of the tip-sample contact 


\section{WILEY-VCH}

will change resulting in a different SThM response. Indeed, $R_{X}$ vs $T_{S}$ curves for probe heater temperature $T_{h 1}=350 \mathrm{~K}$ are shifted downwards with respect to the ones for $T_{h 2}=450 \mathrm{~K}$ (see Figure $2 \mathrm{c})$. The tip-sample contact temperature $\left(T_{S \text {-cont }}\right)$ should be used in Eq.1 instead of $T_{S}$ in the form of $T_{S-c o n t}=\alpha T_{S}+(1-\alpha) T_{h}$ with $\alpha$ being the local heating factor. For the case of SOS and for $a=0.65$ the two curves match as shown in inset of Figure 2b. By applying the same correction for the different 2D structures on $\mathrm{SiO}_{2}, 0.73,0.88,0.88$ were found for $\mathrm{GS}$, MS and MGS areas, respectively (see Supporting Information Figure SI9). This indicates that apart from the cross-plane heat transport from the hot tip to the $\mathrm{SiO}_{2}$ substrate, $\mathrm{SThM}$ also probes the in-plane transport in the 2D material, which depends on their $k$ (see schematic representation Figure 2b).

The sample local heating arises from poor heat conduction in the sample. As heat flows through the material, it builds-up and creates a local temperature rise. The latter is linked to the sample ability to dissipate heat. This dissipation ability can be described by the sample heat conductance often confused with sample conductivity, as described by Wu et al. ${ }^{[36]} \mathrm{We}$ link the higher sample heating observed on the SOS and on the GS compared to the ones on MS and MGS by the different thermal coupling between the 2D materials and the substrate. The graphene $/ \mathrm{SiO}_{2}$ interface thermal resistivity is lower than $\mathrm{MoS}_{2} / \mathrm{SiO}_{2}$. Thus when the probe contacts the graphene, $\mathrm{SiO}_{2}$ has the larger contribution to the $R_{X}$ compared to when the probe is in contact with the $\mathrm{MoS}_{2}$. With graphene, more heat is exchanged with the substrate, which therefore heats up locally. On the contrary, less heat flows from the $\mathrm{MoS}_{2}$ to the $\mathrm{SiO}_{2}$ and the temperature rise of the substrate is therefore smaller.

As mentioned above, $R_{X}$ of the different areas of the sample is linked to the substrate thermal properties. To obtain a better insight into the thermal transport properties of the $2 \mathrm{D}$ structures, we use a diffusive transport model for $R_{s p r}$ of a layer on substrate ${ }^{[37]}$ which already has been shown to be applicable for graphene on $\mathrm{SiO}_{2}{ }^{[20 \mathrm{~b}, 29,38]}$ This model transforms the 


\section{WILEY-VCH}

directional-dependent thermal conductivities of the layer to an effective isotropic thermal conductivity of the layer with thickness larger than the real thickness of the material (See Methods and Supporting Information note 4). By combining these with the Eq. 2, we obtain the effective isotropic thermal conductivities $\left(k_{e f f}\right)$ for particular sample temperatures of the different 2D structures plotted in Figure 3a.

The effective thermal conductivity of graphene $\left(k_{e f f(G)}\right)$ increases with temperature in the range of $150-300 \mathrm{~K}$, in good agreement with previous studies. ${ }^{[18 \mathrm{a}, 18 c, 39]}$ Depending on the sample temperature, the effective thermal conductivity of graphene is suppressed by 6-12 times, by the addition of the top layer of $\mathrm{MoS}_{2}$. On the other hand, $\mathrm{MoS}_{2}$ has a much lower effective thermal conductivity $\left(k_{e f f(M)}\right)$ than graphene with similar behavior for the whole temperature range of the experiment. We believe that non-monotonic dependence of the $k_{e f f}$ for $\mathrm{MoS}_{2}$ and graphene/MoS 2 heterostructure on $T_{S}$ at low temperature is related to the heat transit thermal phenomena in the silicon tip. The absolute effective thermal conductivity difference $\Delta k_{e f f}=k_{e f f(G)}-k_{e f f(G M)}$, and $\Delta k_{e f f}=k_{e f f(G M)}-k_{e f f(M)}$ decreases for the lower temperature regime, which explains the higher thermal image resolution between the corresponding areas at lower temperatures.

Using the same diffusive model we calculate the graphene/MoS 2 boundary thermal resistivity $\left(r_{\operatorname{lnt}(G M)}\right)$, which to the best of our knowledge has not been experimentally reported to date, despite its strong impact on applications of $2 \mathrm{D}$ materials. The calculated $r_{\text {int }(G M)}$ (see Figure 3c) increases with decreasing temperature. Values observed with molecular dynamics (MD) simulations show a similar trend, but with notably higher values. ${ }^{[40]}$ Our values lie in between the values observed for Graphene/hBN and $\mathrm{MoS}_{2} / \mathrm{hBN}^{[41]} \mathrm{vdW}$ heterostructures and higher than ones reported for graphene on diffrerent substrates ${ }^{[42]}$. Furthermore, $r_{\text {int }(G M)}$ is lower than the $\mathrm{SiO}_{2} / \mathrm{MoS}_{2}$ interface thermal resistivity of ref. ${ }^{[30 a]}$, indicating that $\mathrm{MoS}_{2}$ is better attached on graphene than on $\mathrm{SiO}_{2}$, which is also supported by our Ultrasonic Force Microscopy 


\section{WILEY-VCH}

(UFM) nanomechanical characterisation of the these heterostructures (see Supporting Information note 7).

Before proceeding further, we comment on the limitations of the above diffusive model, which as previously reported, ${ }^{[20 \mathrm{~b}]}$ can overestimate or underestimate the calculated $R_{s p r}$, due to the nanoscale tip-sample contact size. More specifically, the reported thermal conductivities and interface thermal resistivities that we use are values for microscopic samples, whereas at the nanoscale, size effects are important and expected to add uncertainties in the calculated values. Furthermore, in all calculations, we use the temperature of the sample measured macroscopically during the experiment. In reality for lower conductivity samples, as we saw for the case of $\mathrm{SiO}_{2}$, the local sample temperature under the tip could be somewhat higher than the macroscopic one. This assumption is expected to add uncertainties to the calculated quantities. For example, we obtain $R_{\text {tip }}$ from measurements on the $\mathrm{SiO}_{2}$ area and we will use this value for the calculation of $k_{\text {eff }}$ of the $2 \mathrm{D}$ materials areas for each temperature, thus assuming that all local temperatures are the same for all areas. Since different regions have different thermal conductances, the local heating caused by the tip will be different. However, as shown above, the local heating effect is highest mostly on the $\mathrm{SiO}_{2}$. Uncertainties for the other regions are notably smaller and are included in the estimation of $R_{t i p}$ as an upper bound (see methods).

To obtain better insight into the suppression of the thermal conductance of GS with the addition of a top layer of $\mathrm{MoS}_{2}$, we used density functional theory (DFT) to find the ground state geometry of graphene and $\mathrm{MoS}_{2}$ unit cells, in agreement with the calculated lattice constants in the literature. ${ }^{[13]}$ In the case of graphene $/ \mathrm{MoS}_{2}$ heterostructures, we find a $1 \%$ lattice mismatch. We use the harmonic approximation ${ }^{[43]}$ to calculate forces on each atom in order to obtain a dynamical matrix and combine it with the Gollum Green's function method to calculate the transmission coefficient $T_{p h}(\hbar \omega)$ of phonons with energy $\hbar \omega$ traversing from the tip to the 


\section{WILEY-VCH}

substrate. ${ }^{[43]}$ The thermal conductance due to phonons is then obtained from $T_{p h}(\hbar \omega)$ using a Landauer-like formula (see methods).

Figure 4a shows the cross-plane phonon transmission coefficient through graphene/MoS2 vdW heterostructures (yellow line) and for Bernal stacked bilayer graphene (blue line) for comparison. Clearly, the amplitude of $T_{p h}$ is much higher in graphene/graphene compared to graphene/MoS2. In addition, due to the lower Debye frequency in $\mathrm{MoS}_{2}$, all modes with energies higher than $\sim 70 \mathrm{meV}$ are filtered. These leads to a significantly lower thermal conductance in graphene/MoS 2 heterostructures by $\sim 3$ orders of magnitude at RT (Figure $4 \mathrm{~b}$ ), in agreement with our measurements (Figure 3a). This is attributed to a large mismatch between the masses of $\mathrm{Mo}, \mathrm{S}$ and $\mathrm{C}$ atoms in graphene/MoS2, compared with the all carbon structure in bilayer graphene.

Due to a weak phonon coupling between top and bottom graphene layers, the transmission coefficient and consequently the thermal conductance is reduced by a factor of ca. 5 for bilayer graphene (blue curve in Figure $4 \mathrm{c}, \mathrm{d}$ ) compared to in-plane monolayer graphene (green curve in Figure $4 \mathrm{c}$,d). Similarly, $T_{p h}$ and $\kappa_{p h}$ is reduced from in-plane monolayer $\mathrm{MoS}_{2}$ (red curve in Figure $4 \mathrm{c}, \mathrm{d}$ ) to bilayer $\mathrm{MoS}_{2}$ (purple curve in Figure $4 \mathrm{c}, \mathrm{d}$ ). However, a highest suppression of heat transport (approx. 3 orders of magnitude) is obtained in graphene/ $\mathrm{MoS}_{2}$ (Figure 4 a,b). This demonstrates that the mismatch between the masses of atoms in graphene/ $\mathrm{MoS}_{2}$ has a larger contribution $(>80 \%)$ to the overall thermal conductance suppression compared to phonon transport filtering in the vdW distance between the layers.

Finally, we compare the experimental effective thermal conductivities with the calculated ones and literature experimental values. Starting from graphene and $\mathrm{MoS}_{2}$, we find that their effective thermal conductivity ratio $k_{e f f(G)} / k_{e f f(M)}$ observed in the experiment is about 10 in a good agreement with the calculations. These ratios are comparable with available literature values for supported graphene and $\mathrm{MoS}_{2}$ at $\mathrm{RT}$, since there are no reported 


\section{WILEY-VCH}

experimental values for a single layer of supported $\mathrm{MoS}_{2}$ at LT. More specifically, $k$ for $\mathrm{SiO}_{2}$ supported $\mathrm{MoS}_{2}$ has been reported between $50-60 \mathrm{Wm}^{-1} \mathrm{~K}^{-1}[11 \mathrm{a}, 17]$ and for graphene around $600 \mathrm{Wm}^{-1} \mathrm{~K}^{-1}$ [18a, 18c] which indeed gives similar ratios. The reduction of thermal conductance of graphene with the addition of a $\mathrm{MoS}_{2}$ layer observed in the theory is much higher than the experiment. Furthermore, in contrast to the experimental result, the theoretical calculations predict a higher thermal conductance of $\mathrm{MoS}_{2}$ compared to that of the graphene/MoS 2 heterostructure. This counter-intuitive behavior is associated with the heat dissipation through in-plane graphene, which acts as an extra heat transfer channel compared to the $\mathrm{MoS}_{2}$ itself. This is also supported by the fact that the heterostructure heats locally less than the $\mathrm{MoS}_{2}$ as demonstrated above. This behaviour is similar to the one observed for increased thermal conductance with the number of graphene layers on $\mathrm{SiO}_{2}$. ${ }^{20 \mathrm{a}, 20 \mathrm{~b}, 44]}$

\section{Conclusions}

In conclusion, an expansion of the nanoscale thermal transport measurements in the SThM to the cryogenic temperatures of the sample was reported. The increased thermal resolution of $\mathrm{SThM}$ at temperatures as low as $150 \mathrm{~K}$ provides a better insight into the thermal transport mechanisms of 2D materials in the nanoscale. Furthermore, we demonstrated that through high thermal and lateral resolution imaging at cryogenic temperatures, we are able to directly observe the contribution of ballistic transport at the edge of the detached areas of exfoliated graphene, which leads to a size dependent thermal conductance. We measured for the first time the thermal conductance of 2D structures including graphene, $\mathrm{MoS}_{2}$ and a graphene/MoS 2 heterostructure, with sub-30nm resolution at various sample temperatures ranging from $150-300 \mathrm{~K}$. In addition, we extracted the interface thermal resistivity between graphene and $\mathrm{MoS}_{2}$. We demonstrate that the addition of a $\mathrm{MoS}_{2}$ layer on top of graphene reduces the thermal conductance by one order of magnitude. This suppression originates from phonon transport filtering due to the weak vdW coupling between the layers and the vibrational mismatch 


\section{WILEY-VCH}

between $\mathrm{MoS}_{2}$ and graphene layers; our results demonstrate that the latter has the larger effect. The study of thermal transport phenomena with sub-30nm resolution in wide temperature range offers the possibility of understanding heat transport phenomena in 2D-nanostructures as we mention in the conclusions, and lead to a better engineering and implementation of such materials at devices.

\section{Experimental Section}

SThM: We use commercially available (Anasys Instruments, AN-300) doped silicon probes (DSi), which have a geometry similar to the standard micromachined Si AFM probes except that the cantilever is formed by two highly doped, and hence, highly electrically conductive, legs. The probe heater that also serves as a temperature sensor is formed by a moderately doped area at the end of the cantilever with a probing sharp tip placed immediately under the heater. With the DC or AC voltage applied to the probe, the heater can be heated to the desired temperature $\left(T_{h}\right)$ usually few tens of $\mathrm{K}$ above the temperature of the microscope, with the latter defining the temperature of the cantilever base $\left(T_{M}\right)$. The probe forms one leg of a balanced Wheatstone bridge operating at $91 \mathrm{kHz}$, with the bridge output amplified via a low noise instrumental amplifier, producing the raw SThM signal used for the thermal images and heat transport measurements. This signal is proportional to the probe electrical resistance, and hence is a measure of the heater temperature, with sensitivity down to $5 \times 10^{-3} \mathrm{~K}$. The calibration of the SThM signal and its electrical resistance versus temperature is described elsewhere. ${ }^{[26]}$ When the tip is in contact with a sample, the temperature of the heater changes depending on the heat transfer between the tip and the surface.

Cryo-SThM measurements: All experiments were performed in high vacuum $\left(5 \times 10^{-8}-1 \times\right.$ $10^{-6}$ Torr). By adding $\mathrm{LN}_{2}$ in the external Dewar, the copper braid, the sample holder and, 


\section{WILEY-VCH}

subsequently, the sample attached to the sample holder with a low temperature compatible adhesive began to cool down. As the braid and sample holder temperatures decrease, they act as cryo-pump and any residual moisture or contamination will condensate on cold surfaces. To avoid this effect on the sample, it is kept at elevated temperature of about $300 \mathrm{~K}$ for 1 hour by the dedicated heater, while the braid is cooling down. During the whole experiment, the sample and the microscope temperatures $T_{S}, T_{M}$ are monitored. The lowest temperature that the sample reaches in such setup is typically $150 \mathrm{~K}$. This temperature can be increased up to the room temperature $(300 \mathrm{~K})$ and controlled through integrated resistive heaters on the sample holder. For each temperature we record a thermal image approach and retract curves of SThM signal.

Graphene-MoS $\mathrm{S}_{2}$ heterostructure Fabrication: The graphene- $\mathrm{MoS}_{2}$ heterostructure was fabricated by a pick-and-place method that allows to pick-up an atomically-thin flake from a substrate with the subsequent transfer on another substrate. ${ }^{[45]}$ Graphene and $\mathrm{MoS}_{2}$ were exfoliated by standard scotch tape method on a $\mathrm{SiO}_{2}$ (280 nm on $\mathrm{Si}$ wafer) substrate and the atomically-thin flakes were identified by optical microscopy. The $\mathrm{MoS}_{2}$ monolayer was then picked-up and transferred on top of the graphene flake, ensuring a clean interface between the two materials (see Supporting Information note 7 for more information). In addition, few graphene flakes were exfoliated on a second $\mathrm{SiO}_{2}$ substrate.

Diffusive model for spreading resistance: The spreading resistance of a heat source of diameter $d_{0}$ on a layer with effective thickness $t_{e f f}$ on a substrate is given by:

$R_{s p r}=\frac{1}{\pi k_{e f f} d_{0} / 2} \int_{0}^{\infty}\left[\frac{1+K \exp \left(-2 \zeta^{t_{e f f}} / d_{0} / 2\right)}{1-\operatorname{Kexp}\left(-2 \zeta^{t_{e f f}} / d_{0} / 2\right)}\right] J_{1}(\zeta) \sin \zeta \frac{d \zeta}{\zeta^{2}}$

Where $J_{1}(\zeta)$ is the Bessel function, $K=\left(1-k_{\text {sub }} / k_{\text {eff }}\right) /\left(1+k_{\text {sub }} / k_{\text {eff }}\right)$ with $k_{\text {sub }}$ being the thermal conductivity of the substrate and $k_{\text {eff }}$ the effective thermal conductivity of the 2D 


\section{WILEY-VCH}

material of effective thickness $t_{\text {eff }}=n t+r_{\text {int }} k_{\text {eff }}$ with $t$ being the thickness of the 2D material, $n$ the number of layers, and $r_{i n t}$ the thermal interface resistivity between the substrate and the 2D material.

The $R_{s p r}$ is substituted to Eq. 2 of the main text and we get the $k_{\text {eff }}$ of graphene $\left(k_{e f f(G)}\right), \mathrm{MoS}_{2}\left(k_{e f f(M)}\right)$ and GM heterostructure $\left(k_{e f f(G M)}\right)$. As $k_{s u b}$ we use the temperature dependent thermal conductivity of $\mathrm{SiO}_{2}\left(k_{\mathrm{SiO} 2}\right) \cdot{ }^{[32]} R_{\text {int }}$ between the DSi tip covered with native $\mathrm{SiO}_{2}$ and the 2D material can be defined as $R_{\text {int (S2D) }}=r_{i n t(S 2 D)} /\left(\pi d_{0}^{2} / 4\right)^{[20 \mathrm{~d}, 25]}$ with $r_{i n t(S 2 D)}$ being the thermal contact resistivity of $\mathrm{SiO}_{2} /$ graphene $\left(r_{\text {int }(G S)}\right)$ and $\mathrm{SiO}_{2} / \mathrm{MoS}_{2}$ $\left(r_{\text {int }(M S)}\right)$ from ref ${ }^{[30 \mathrm{a}]}$. For the graphene and $\mathrm{MoS}_{2}$ single layers we directly apply this model while for the graphene/MoS 2 heterostructure, we assume an isotropic layer consisting of graphene and $\mathrm{MoS}_{2}$ with $t_{\text {eff }}=t_{M}+t_{G}+r_{\text {int }(G S)} k_{e f f(G M)}$ and $k_{\text {eff }}=k_{\text {eff(GM) }}$. We estimate an upper bound of $R_{\text {tip }}$ from the thermal contact resistance measured on the $\mathrm{SiO}_{2}\left(R_{X S}\right)$, by using Eq. 2 with $R_{s p r}$ calculated from Eq.3 for an isotropic $\mathrm{SiO}_{2}$ layer on $\mathrm{Si}$ with $k_{e f f}$ being the thermal conductivity of bulk $\mathrm{SiO}_{2}, t_{\text {eff }}=280 \mathrm{~nm}$ and by setting $R_{\text {int }}$ between the $\mathrm{SiO}_{2}$ sample and $\mathrm{SiO}_{2}$ covered DS tip to zero $\left(R_{\text {int }(\text { Stip })}=0\right)$.

For the calculation of the graphene/ $\mathrm{MoS}_{2}$ interface thermal resistivity the graphene/MoS 2 heterostructure is modeled as a $\mathrm{MoS}_{2}$ isotropic layer on top of $\mathrm{SiO}_{2}$ with an effective thickness $t_{(e f f-G M)}=t_{M}+r_{\text {int }(e f f-G M)} k_{e f f(M)}$ where $r_{i n t(e f f-G M)}$ is the effective thermal resistivity of the $\mathrm{MoS}_{2}$ and $\mathrm{SiO}_{2}$. It includes $k_{\text {eff }(M)}$ and the interfaces resistance of graphene $/ \mathrm{SiO}_{2}$ and graphene/MoS 2 , in the form $r_{i n t(e f f-M G)}=\frac{t_{e f f(G)}}{k_{e f f}(G)}+r_{i n t(G M)}$. As $k_{e f f(M)}$ and $k_{e f f(G)}$ we use the values obtained for $\mathrm{MoS}_{2}$ and graphene single layers respectively. All temperature depended values from literature are according to the macroscopic sample temperature. 


\section{WILEY-VCH}

Computational Methods - Geometry optimization: The geometry of each structure was relaxed to the force tolerance of $10 \mathrm{meV} / \AA \AA$ using the SIESTA ${ }^{[46]}$ implementation of density functional theory (DFT), with a double- $\zeta$ polarized basis set (DZP) and the Generalized Gradient Approximation (GGA) functional with Perdew-Burke-Ernzerhof (PBE) parameterization. A real-space grid was defined with an equivalent energy cut-off of $250 \mathrm{Ry}$.

Computational Methods - Thermal transport: Following the method described in ${ }^{[43,47]}$ a set of xyz coordinates were generated by displacing each atom from the relaxed xyz geometry in the positive and negative $\mathrm{x}, \mathrm{y}$ and $\mathrm{z}$ directions with $\delta q^{\prime}=0.01 \AA$. The forces $F_{i}^{q}=\left(F_{i}^{x}, F_{i}^{y}, F_{i}^{z}\right)$ in three directions $q_{i}=\left(x_{i}, y_{i}, z_{i}\right)$ on each atom were then calculated and used to construct the dynamical matrix $D_{i j}=K_{i j}^{q q \prime} / M_{i j}$ where the mass matrix $M=\sqrt{M_{i} M_{j}}$ and $K_{i j}^{q q \prime}=$ $\left[F_{i}^{q}\left(\delta q_{j}^{\prime}\right)-F_{j}^{q}\left(-\delta q_{j}^{\prime}\right)\right] / 2 \delta q_{j}^{\prime}$ for $i \neq j$ obtained from finite differences. To satisfy momentum conservation, $K$ for $i=j$ (diagonal terms) is calculated from $K_{i i}=-\sum_{i \neq j} K_{i j}$. The phonon transmission $T(\omega)$ then can be calculated from the relation $T(\omega)=$ $\operatorname{Trace}\left(\Gamma_{L}(\omega) G^{R}(\omega) \Gamma_{R}(\omega) G^{R \dagger}(\omega)\right)$ where $\Gamma_{L, R}(\omega)=i\left(\sum_{L, R}(\omega)-\sum_{L, R}^{\dagger}(\omega)\right)$ describes the level broadening due to the coupling to the left $\mathrm{L}$ and right $\mathrm{R}$ electrodes, $\sum_{L, R}(\omega)$ are the retarded self-frequencies associated with this coupling and $G^{R}=\left(\omega^{2} I-\mathrm{D}-\sum_{L}-\sum_{R}\right)^{-1}$ is the retarded Green's function, where D and I are the dynamical and the unit matrices, respectively. The phonon thermal conductance $\kappa_{p h}$ at temperature $T$ is then calculated from $\kappa_{p h}(T)=\frac{1}{2 \pi} \int_{0}^{\infty} \hbar \omega \mathrm{T}(\omega) \frac{\partial f_{B E}(\omega, T)}{\partial T} d \omega$ where $f_{B E}(\omega, T)=\left(e^{\hbar \omega / k_{B} T}-1\right)^{-1}$ is Bose-Einstein distribution function and $\hbar$ is reduced Planck's constant and $k_{B}=8.6 \times 10^{-5} \mathrm{eV} / \mathrm{K}$ is Boltzmann's constant.

\section{Supporting Information}




\section{WILEY-VCH}

Supporting Information is available from the Wiley Online Library or from the author.

\section{Acknowledgements}

C.E., J.S. and O.K. contributed equally to the paper. Authors acknowledge helpful scientific support and discussion of B. Robinson, S. Gomez, A. Robson and V. Tsepelin. O.K., C.E. and J.S. acknowledge the support of the EU grant QUANTIHEAT (project \# 604668). H.S. and S.S. acknowledge the Leverhulme Trust (Leverhulme Early Career Fellowships no. ECF-2017-186 and ECF-2018-375) for funding. This work was supported by EPSRC grants EP/K023373/1, EP/G015570/1, EP/P027156/1, EP/M013243/1, EP/N03337X/1, EP/N017188/1 and the ECH2020 FET Open project 767187 "QuIET" and the EU project Bac-to-Fuel. M. M. acknowledges support of Lancaster University FST grant and A.J.M.-M. acknowledges financial support from the European Commission (Marie Sklodowska-Curie Individual Fellowships, grant ID 791536). T. M. and AJM.-M. acknowledge financial support by the European Union (grant agreement No. 785219 Graphene Flagship).

Received: ((will be filled in by the editorial staff))

Revised: ((will be filled in by the editorial staff)) Published online: ((will be filled in by the editorial staff))

References
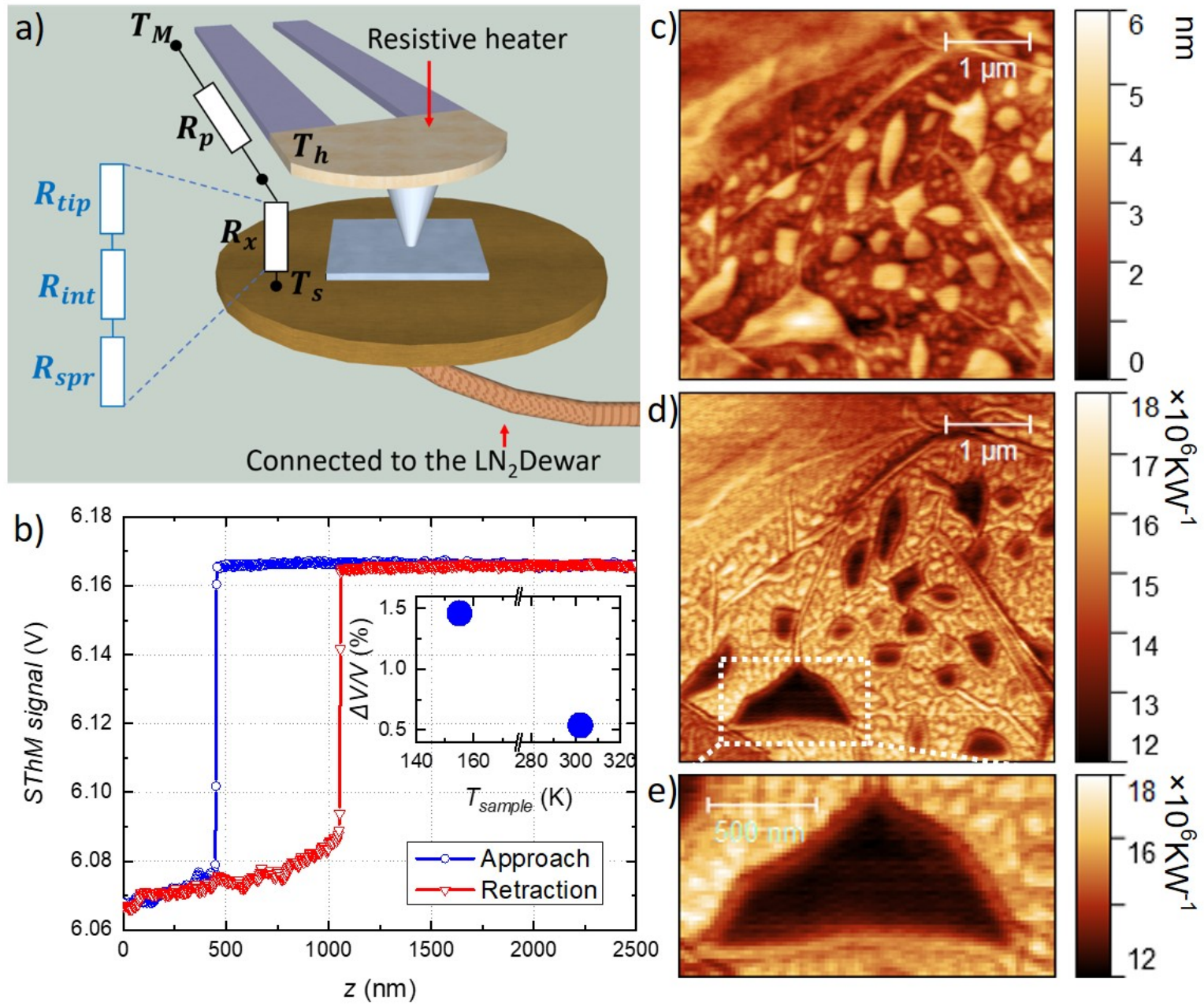


\section{WILEY-VCH}

Figure 1. (a) Schematic representation of the high vacuum cryo-SThM with probe tip in contact with the sample. The sample holder is connected with a copper braid to a $\mathrm{LN}_{2}$ Dewar. $T_{S}, T_{M}$ and $T_{h}$ are the sample, probe base and heater temperatures, respectively. (b) Approach (blue) and retract (red) curves for thermal response acquired on $280 \mathrm{~nm} \mathrm{SiO}_{2}$ on $\mathrm{Si}$ sample $\left(T_{h}=\right.$ $\left.350 \mathrm{~K}, T_{S}=155 \mathrm{~K}\right)$. Inset: The relative signal "jump" $\frac{\Delta V}{V}=\frac{V_{n c}-V_{c}}{V_{n c}}$ for $\mathrm{SiO}_{2}$ on $\mathrm{Si}$ substrate at two different sample temperatures. (c-e) Topography (c) and thermal resistance (d) images of few layers graphene on $\mathrm{SiO}_{2}$ simultaneously acquired at $T_{S}=210 \mathrm{~K}$. A zoom of the thermal resistance image is shown (e) as well.

a)

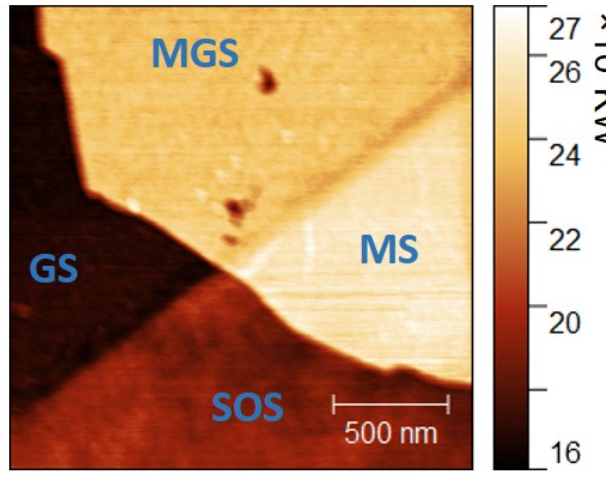

b)

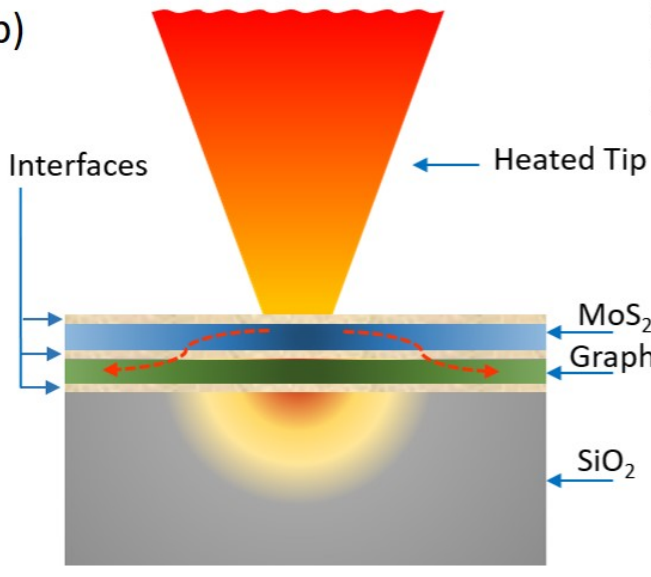

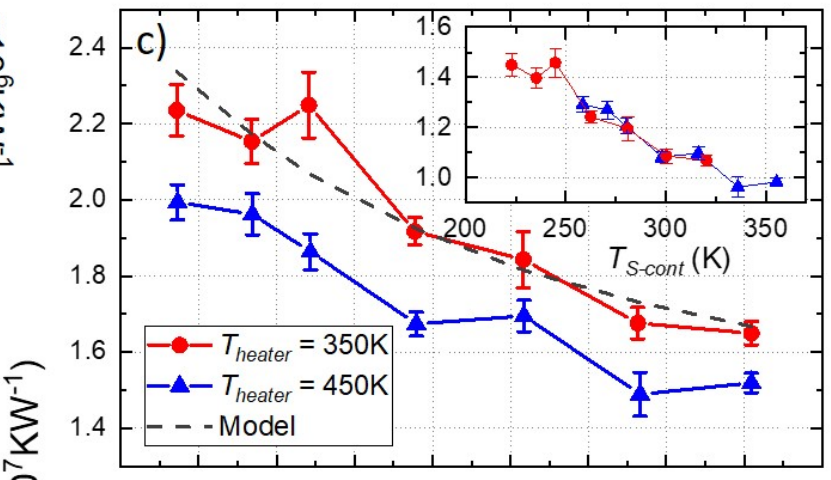

을

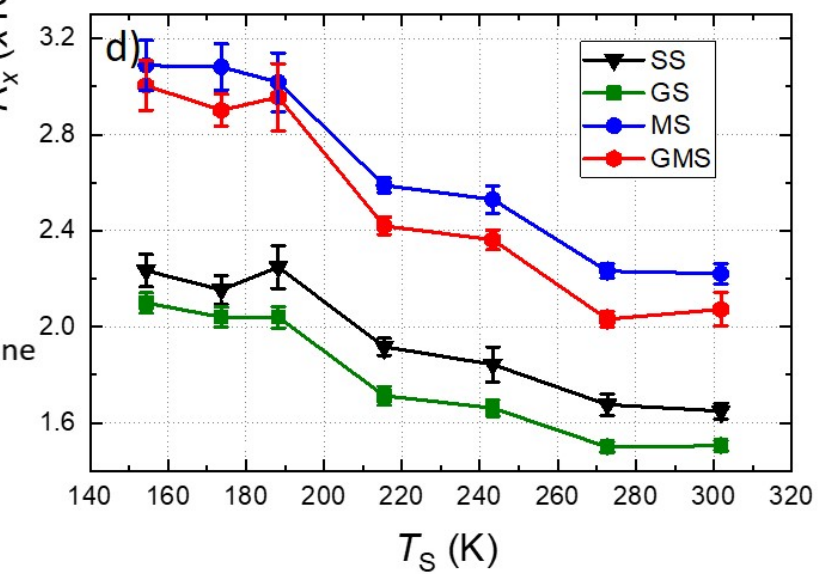




\section{WILEY-VCH}

Figure 2. (a) $R_{X}$ image acquired at $T_{S}=220 \mathrm{~K}$ with the different areas of interest. Note that this image is taken with a probe of different contact radius than the one in Figure 1d and e (b) Schematic representation of the heat transport from the tip to the substrate through the $2 \mathrm{D}$ structures. (c) $R_{X}$ of DSi tip in contact with SOS sample at various sample temperatures with the tip heater temperature at $350 \mathrm{~K}$ (red) and $450 \mathrm{~K}$ (blue) (dashed line is the fitted curve). Inset: $R_{X}$ versus tip-sample contact temperature after applying the correction to temperature due to local heating by the tip. (d) $R_{X}$ of the different areas as extracted from the images for sample temperature range of $150-300 \mathrm{~K}$.

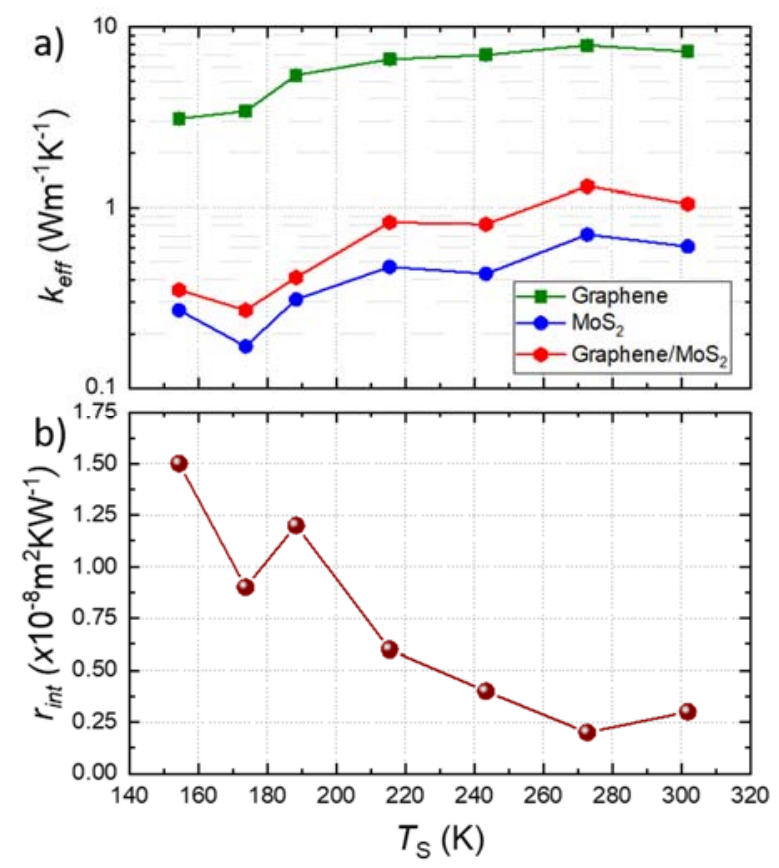

Figure 3. (a) Effective thermal conductivity $k_{e f f}$ as calculated from the diffusive model (see Methods) for the different areas of the image as a function of sample temperature. (b) graphene/MoS 2 Interface thermal resistivity. 
WILEY-VCH
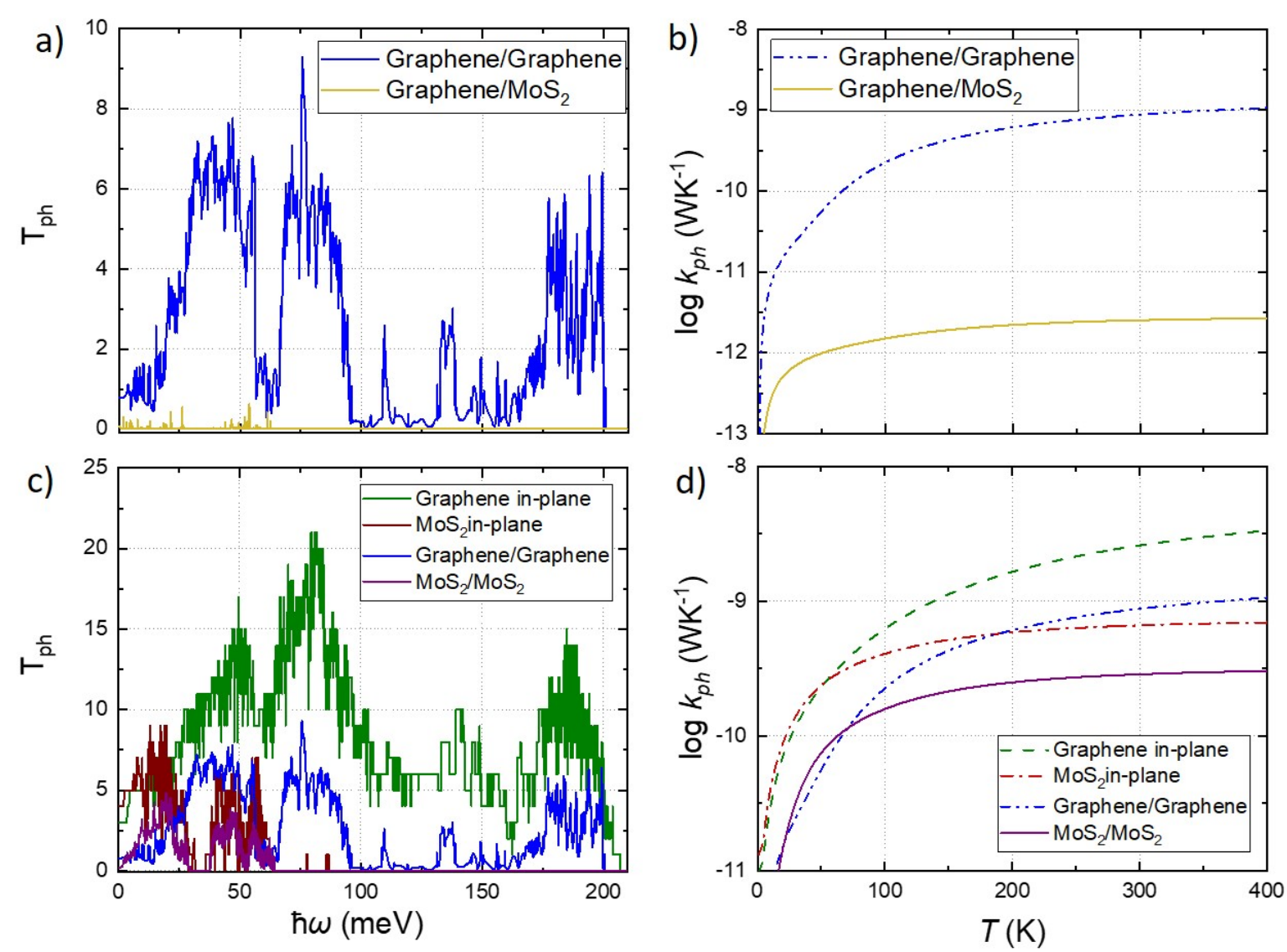

Figure 4. $(\mathrm{a}, \mathrm{b})$ Thermal transport through cross-plane graphene and graphene/ $\mathrm{MoS}_{2}$ heterostructures. (a) Transmission coefficient for phonons with energies $\hbar \omega$ passing through graphene/graphene and graphene/MoS 2 VdW junctions. (b) Phonon thermal conductance of graphene/graphene and graphene/MoS2. (c,d) In-plane and cross-plane thermal transport through graphene, $\mathrm{MoS}_{2}$, graphene/graphene, $\mathrm{MoS}_{2} / \mathrm{MoS}_{2}$ (c) Transmission coefficient for phonons with energies $\hbar \omega$ and (d) Phonon thermal conductance. 


\section{WILEY-VCH}

Nanothermal properties of 2D nanostructures with sub-30nm resolution from $150 \mathrm{~K}$ to room temperature are probed by Scanning Thermal Microscopy. By engineering the thermal conductance, either with the use of thermal anisotropy of the in-plane and cross-plane thermal conductivities or through ballistic transport phenomena we can notably decrease the thermal conductance which is expected to lead to more efficient thermoelectric devices.

\section{Keyword}

C. Evangeli*, J. Spiece, H. Sadeghi*, S. Sangtarash, A. J. Molina-Mendoza, M. Mucientes, T. Mueller, C. Lambert and O. Kolosov*

Nanoscale thermal transport in 2D nanostructures from cryogenic to room temperatures

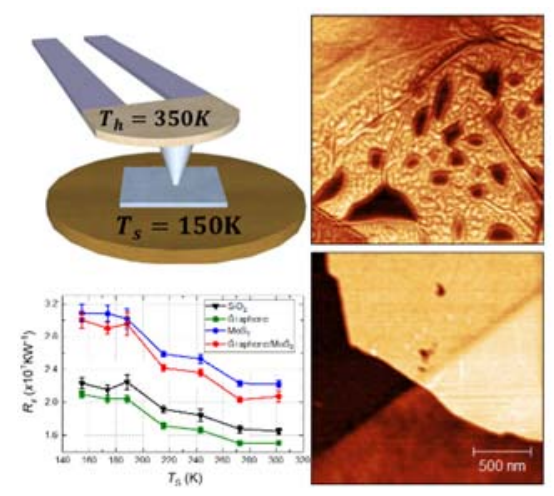

ToC figure ((Please choose one size: $55 \mathrm{~mm}$ broad $\times 50 \mathrm{~mm}$ high or $110 \mathrm{~mm}$ broad $\times 20 \mathrm{~mm}$ high. Please do not use any other dimensions)) 


\section{WILEY-VCH}

((Supporting Information can be included here using this template))

Copyright WILEY-VCH Verlag GmbH \& Co. KGaA, 69469 Weinheim, Germany, 2016.

\section{Supporting Information}

Title ((no stars))

Author(s), and Corresponding Author(s)* ((write out full first and last names))

((Please insert your Supporting Information text/figures here. Please note: Supporting Display items, should be referred to as Figure S1, Equation S2, etc., in the main text...)

[1] H. X. Han, Y. Zhang, N. Wang, M. K. Samani, Y. X. Ni, Z. Y. Mijbil, M. Edwards, S. Y. Xiong, K. Saaskilahti, M. Murugesan, Y. F. Fu, L. L. Ye, H. Sadeghi, S. Bailey, Y. A. Kosevich, C. J. Lambert, J. Liu, S. Volz, Nature Communications 2016, 7.

[2] a) A. A. Balandin, Nat Mater 2011, 10, 569; b) J. Renteria, D. Nika, A. Balandin, Applied Sciences 2014, 4, 525; c) H. Song, J. Liu, B. Liu, J. Wu, H.-M. Cheng, F. Kang, Joule 2018, 2, 442; d) Z. Zhang, S. Hu, J. Chen, B. Li, Nanotechnology 2017, 28, 225704.

[3] a) R. Cheng, S. Jiang, Y. Chen, Y. Liu, N. Weiss, H. C. Cheng, H. Wu, Y. Huang, X. Duan, Nat Commun 2014, 5, 5143; b) B. Radisavljevic, A. Radenovic, J. Brivio, V. Giacometti, A. Kis, Nat Nanotechnol 2011, 6, 147.

[4] C. Chiritescu, D. G. Cahill, N. Nguyen, D. Johnson, A. Bodapati, P. Keblinski, P. Zschack, Science 2007, 315, 351.

[5] K. S. Novoselov, A. Mishchenko, A. Carvalho, A. H. Castro Neto, Science 2016, 353, aac9439.

[6] M.-L. Tsai, M.-Y. Li, J. R. D. Retamal, K.-T. Lam, Y.-C. Lin, K. Suenaga, L.-J. Chen, G. Liang, L.-J. Li, J.-H. He, Adv. Mater. 2017, 29, 1701168.

[7] N. Gao, X. Fang, Chem. Rev. 2015, 115, 8294.

[8] T. A. Amollo, G. T. Mola, M. S. K. Kirui, V. O. Nyamori, Crit. Rev. Solid State Mater. Sci. 2017, 43, 133.

[9] a) G. Zhang, Y.-W. Zhang, Journal of Materials Chemistry C 2017, 5, 7684; b) M. Buscema, M. Barkelid, V. Zwiller, H. S. van der Zant, G. A. Steele, A. CastellanosGomez, Nano Lett. 2013, 13, 358; c) J. Wu, H. Schmidt, K. K. Amara, X. Xu, G. Eda, B. Ozyilmaz, Nano Lett. 2014, 14, 2730.

[10] a) E. Pop, V. Varshney, A. K. Roy, MRS Bull. 2012, 37, 1273; b) R. Yan, J. R. Simpson, S. Bertolazzi, J. Brivio, M. Watson, X. Wu, A. Kis, T. Luo, A. R. Hight Walker, H. G. Xing, ACS Nano 2014, 8, 986; c) S. Chen, Q. Wu, C. Mishra, J. Kang, H. Zhang, K. Cho, W. Cai, A. A. Balandin, R. S. Ruoff, Nat Mater 2012, 11, 203.

[11] a) Z. Ding, J. W. Jiang, Q. X. Pei, Y. W. Zhang, Nanotechnology 2015, 26, 065703; b) Y. Ni, Y. Chalopin, S. Volz, Appl. Phys. Lett. 2013, 103; c) Y. X. Ni, Y. Chalopin, S. Volz, Appl. Phys. Lett. 2013, 103, 061906. 


\section{WILEY-VCH}

[12] a) D. Olaya, M. Hurtado-Morales, D. Gómez, O. A. Castañeda-Uribe, Z.-Y. Juang, Y. Hernández, $2 D$ Materials 2017, 5; b) C.-C. Chen, Z. Li, L. Shi, S. B. Cronin, Nano Research 2014, 8, 666.

[13] H. Sadeghi, S. Sangtarash, C. J. Lambert, 2D Materials 2017, 4, 015012.

[14] a) K. H. Park, U. Ravaioli, J. Appl. Phys. 2017, 122; b) C. Wang, Y. Liu, L. Li, H. Tan, Nanoscale 2014, 6, 5703.

[15] S. Hu, Z. Zhang, P. Jiang, J. Chen, S. Volz, M. Nomura, B. Li, J Phys Chem Lett 2018, 9, 3959.

[16] A. Harzheim, J. Spiece, C. Evangeli, E. McCann, V. Falko, Y. Sheng, J. H. Warner, G. A. D. Briggs, J. A. Mol, P. Gehring, O. V. Kolosov, Nano Letters 2018, DOI: 10.1021/acs.nanolett.8b03406.

[17] A. Taube, J. Judek, A. Lapinska, M. Zdrojek, ACS Appl Mater Interfaces 2015, 7, 5061. [18] a) M. H. Bae, Z. Li, Z. Aksamija, P. N. Martin, F. Xiong, Z. Y. Ong, I. Knezevic, E. Pop, Nat Commun 2013, 4, 1734; b) M. M. Sadeghi, I. Jo, L. Shi, Proc Natl Acad Sci U $S$ A 2013, 110, 16321; c) J. H. Seol, I. Jo, A. L. Moore, L. Lindsay, Z. H. Aitken, M. T. Pettes, X. Li, Z. Yao, R. Huang, D. Broido, N. Mingo, R. S. Ruoff, L. Shi, Science 2010, 328, 213; d) I. Jo, M. T. Pettes, E. Ou, W. Wu, L. Shi, Appl. Phys. Lett. 2014, 104, 4; e) X. Xu, J. Chen, B. Li, J Phys Condens Matter 2016, 28, 483001.

[19] A. Majumdar, Annu. Rev. Mater. Sci. 1999, 29, 505.

[20] a) M. E. Pumarol, M. C. Rosamond, P. Tovee, M. C. Petty, D. A. Zeze, V. Falko, O. V. Kolosov, Nano Lett. 2012, 12, 2906; b) F. Menges, H. Riel, A. Stemmer, C. Dimitrakopoulos, B. Gotsmann, Phys. Rev. Lett. 2013, 111, 205901; c) P. D. Tovee, O. V. Kolosov, Nanotechnology 2013, 24, 465706; d) S. Gomès, A. Assy, P.-O. Chapuis, physica status solidi (a) 2015, 212, 477.

[21] W. Jeong, K. Kim, Y. Kim, W. Lee, P. Reddy, Scientific Reports 2014, 4, 4975.

[22] a) N. Leconte, H. Kim, H.-J. Kim, D. H. Ha, K. Watanabe, T. Taniguchi, J. Jung, S. Jung, Nanoscale 2017, 9, 6041; b) R. Mazzocco, B. J. Robinson, C. Rabot, A. Delamoreanu, A. Zenasni, J. W. Dickinson, C. Boxall, O. V. Kolosov, Thin Solid Films 2015, 585, 31; c) E. Khestanova, F. Guinea, L. Fumagalli, A. K. Geim, I. V. Grigorieva, Nature Communications 2016, 7, 12587.

[23] N. Mosso, U. Drechsler, F. Menges, P. Nirmalraj, S. Karg, H. Riel, B. Gotsmann, Nat Nano 2017, 12, 430.

[24] J. Spiece, C. Evangeli, K. Lulla, A. Robson, B. Robinson, O. Kolosov, J. Appl. Phys. 2018, 124.

[25] B. Gotsmann, M. A. Lantz, A. Knoll, U. Dürig, in Nanotechnology, DOI: 10.1002/9783527628155.nanotech066, Wiley-VCH Verlag GmbH \& Co. KGaA 2010.

[26] P. Tovee, M. Pumarol, D. Zeze, K. Kjoller, O. Kolosov, J. Appl. Phys. 2012, 112, 114317.

[27] a) T. Georgiou, L. Britnell, P. Blake, R. V. Gorbachev, A. Gholinia, A. K. Geim, C. Casiraghi, K. S. Novoselov, Appl. Phys. Lett. 2011, 99; b) K. Yue, W. Gao, R. Huang, K. M. Liechti, J. Appl. Phys. 2012, 112.

[28] X. Xu, L. F. Pereira, Y. Wang, J. Wu, K. Zhang, X. Zhao, S. Bae, C. Tinh Bui, R. Xie, J. T. Thong, B. H. Hong, K. P. Loh, D. Donadio, B. Li, B. Ozyilmaz, Nat Commun 2014, 5, 3689.

[29] G. Hwang, O. Kwon, Nanoscale 2016, 8, 5280.

[30] a) P. Yasaei, C. J. Foss, K. Karis, A. Behranginia, A. I. El-Ghandour, A. Fathizadeh, J. Olivares, A. K. Majee, C. D. Foster, F. Khalili-Araghi, Z. Aksamija, A. Salehi-Khojin, Advanced Materials Interfaces 2017, 4, 1700334; b) E. Yalon, O. B. Aslan, K. K. H. Smithe, C. J. McClellan, S. V. Suryavanshi, F. Xiong, A. Sood, C. M. Neumann, X. Xu, K. E. Goodson, T. F. Heinz, E. Pop, ACS Appl Mater Interfaces 2017, 9, 43013; c) H. Zhang, C. Y. Hua, D. Ding, A. J. Minnich, Scientific Reports 2015, 5, 5. 


\section{WILEY-VCH}

[31] a) Z. Chen, W. Jang, W. Bao, C. N. Lau, C. Dames, Appl. Phys. Lett. 2009, 95, 161910;

b) K. F. Mak, C. H. Lui, T. F. Heinz, Appl. Phys. Lett. 2010, 97, 3.

[32] M. Anis-ur-Rehman, A. Maqsood, Int. J. Thermophys. 2003, 24, 867.

[33] K. E. Goodson, M. I. Flik, L. T. Su, D. A. Antoniadis, J. Heat Transfer 1994, 116, 317.

[34] J. Chen, G. Zhang, B. W. Li, J. Appl. Phys. 2012, 112, 064319.

[35] C. J. Glassbrenner, G. A. Slack, Phys. Rev. 1964, 134, A1058.

[36] X. Wu, V. Varshney, J. Lee, Y. Pang, A. K. Roy, T. Luo, Chem. Phys. Lett. 2017, 669, 233.

[37] M. M. Yovanovich, J. R. Culham, P. Teertstra, IEEE Transactions on Components, Packaging, and Manufacturing Technology: Part A 1998, 21, 168.

[38] M. M. Sadeghi, S. Park, Y. Huang, D. Akinwande, Z. Yao, J. Murthy, L. Shi, J. Appl. Phys. 2016, 119, 235101.

[39] Y. Xu, Z. Li, W. Duan, Small 2014, 10, 2182.

[40] a) B. Liu, F. M. Meng, C. D. Reddy, J. A. Baimova, N. Srikanth, S. V. Dmitriev, K. Zhou, Rsc Advances 2015, 5, 29193; b) Z. Ding, Q.-X. Pei, J.-W. Jiang, W. Huang, Y.W. Zhang, Carbon 2016, 96, 888.

[41] Y. Liu, Z. Y. Ong, J. Wu, Y. Zhao, K. Watanabe, T. Taniguchi, D. Chi, G. Zhang, J. T. Thong, C. W. Qiu, K. Hippalgaonkar, Sci Rep 2017, 7, 43886.

[42] P. Yasaei, A. Behranginia, Z. Hemmat, A. I. El-Ghandour, C. D. Foster, A. SalehiKhojin, 2D Materials 2017, 4, 035027.

[43] H. Sadeghi, Nanotechnology 2018, 29, 373001.

[44] W. Jang, Z. Chen, W. Bao, C. N. Lau, C. Dames, Nano Lett. 2010, 10, 3909.

[45] F. Pizzocchero, L. Gammelgaard, B. S. Jessen, J. M. Caridad, L. Wang, J. Hone, P. Boggild, T. J. Booth, Nat Commun 2016, 7, 11894.

[46] M. S. José, A. Emilio, D. G. Julian, G. Alberto, J. Javier, O. Pablo, S.-P. Daniel, J. Phys.: Condens. Matter 2002, 14, 2745.

[47] a) H. Sadeghi, S. Sangtarash, C. J. Lambert, Nano Lett. 2015, 15, 7467; b) J. Ferrer, C. J. Lambert, V. M. García-Suárez, D. Z. Manrique, D. Visontai, L. Oroszlany, R. Rodríguez-Ferradás, I. Grace, S. W. D. Bailey, K. Gillemot, H. Sadeghi, L. A. Algharagholy, New Journal of Physics 2014, 16. 


\section{Supporting Information}

\section{Nanoscale thermal transport in 2D}

\section{nanostructures at cryogenic temperatures}

Charalambos Evangeli ${ }^{\dagger, t, * \ddagger}+$ Jean Spiecet, + , Hatef Sadeghi ${ }^{\dagger, *}$, Sara Sangtarash $t$, Aday J.

Molina-Mendoza $\neq$, Marta San Juan Mucientest, Thomas Mueller $\neq$, Colin Lambert ${ }^{\dagger}$ and

$$
\text { Oleg Kolosovt,*¥ }
$$

†Physics Department, Lancaster University, Lancaster, UK.

‡Institute of Photonics, Vienna University of Technology, Gusshausstrasse 27-29, A-1040

Vienna, Austria.

\$These authors contributed equally to the paper 


\section{Contents:}

1. Thermal resistance model for cryogenic measurements

2. Heat transport in the doped Si (DSi) SThM probe tip

3. Sample Temperature control

4. Thermal imaging speed and thermal drift

5. Error Analysis

6. Thermal resistance of $280 \mathrm{~nm} \mathrm{SiO}_{2}$ layer on $\mathrm{Si}$

7. Anisotropy of the thermal transport in the layered system

8. Lateral Resolution of the thermal images in SThM

9. Thermal Resistance Measurements on Si and thermal transport in the DSi

10. Graphene/MoS $\mathbf{S}_{2}$ Heterostructure

11. Sample local heating by the tip

12. SThM imaging of detached Graphene Areas

\section{Thermal resistance model for cryogenic measurements}

When the probe is out-of-contact with the sample, the power applied to the heater gives rise to an excess temperature, $\Delta T$, with respect the probe base temperature, $T_{M}$, that relates to the SThM output voltage by a calibration factor $b, \Delta T=b V$. In vacuum, the only heat transfer channel is through the probe and cantilever themselves and $R_{p}$ is their thermal resistance. When the probe is brought into contact with a sample, a new heat transfer channel is opened going from the heater to the sample heat sink with thermal resistance given by $R_{x}$.

The heat generated in the probe heater, $Q_{h}$, for the just in- and out-of-contact case is given by $Q_{h}=\left(T_{h}^{n c}-T_{M}\right) / R_{p}$,

$Q_{h}=\left(T_{h}^{c}-T_{M}\right) / R_{p}+\left(T_{h}^{c}-T_{S}\right) / R_{X}$, 
respectively. Where $T_{h}^{c}$ and $T_{h}^{n c}$ are the temperatures of the heater for the in- and out-of-contact case respectively, and $T_{S}$ is the sample temperature. For both in- and out-of-contact cases, we are not measuring absolute temperatures but only excess temperatures with respect to the temperature at which the bridge balance was realized which is the same as $T_{M}$. Therefore,

$$
\Delta T_{c, n c}=T_{h}^{c, n c}-T_{M}=b V_{c, n c} .
$$

By bringing the probe in and out of contact while its temperature is recorded and using eq. S1,

S2 and S3, we obtain the normalized temperature change between out and in contact:

$$
\frac{\Delta T_{n c}-\Delta T_{c}}{\Delta T_{n c}}=\frac{V_{n c}-V_{c}}{V_{n c}}=\frac{T_{h}^{n c}-T_{h}^{c}}{T_{h}^{c, n c}-T_{M}}=\frac{1}{R_{p}+R_{X}}\left(R_{p}+\frac{T_{M}-T_{S}}{Q_{h}}\right) .
$$

\section{Heat transport in the doped Si (DSi) SThM probe tip}

\subsection{Temperature distribution along the tip}

For the modelling of the temperature distribution in the doped silicon conical tip, we assume: a truncated conical tip of known apex radius, $r_{0}$, half-angle and length $l_{t}$, diffusive transport in the whole tip, Power $Q$ flowing uniformly through the tip. The heat equation can be written in the case of $1 \mathrm{D}$ heat conduction as :

$Q=-k A(l) \frac{d T}{d l}$

where $Q$ is the rate of heat conduction, $k$ is the thermal conductivity of the material and $A(l)$ is the section of the cone of the at the position $l$ (see Figure S1a).

From trigonometry, we have $\tan \alpha=\frac{r_{0}}{l_{0}}=\frac{r}{l+l_{0}}$ and thus $A(l)=\pi r^{2}$.

In order to get the temperature distribution in the tip, we integrate the heat equation

$\int_{T=T_{0}}^{T(l)} d T=\int_{l=0}^{l}-\frac{Q}{\pi k\left(\left(l_{t}-l\right) \tan \alpha+r_{0}\right)^{2}} d l$.

This gives

$T(l)=T_{0}-\frac{Q\left(l-l_{t}\right)}{\left(l_{t} \tan \alpha+r_{0}\right) \pi k\left(l \tan \alpha+r_{0}\right)}$, 
with $T_{0}$ being the temperature at the heater side.

By defining $Q$ from the heat flowing from the heater to the sample through the whole tip:

$Q=\frac{\left(T_{h}-T_{S}\right) r_{0} \pi k\left(l \tan \alpha+r_{0}\right)}{l_{t}}$,

we can plot the temperature distribution within the conical silicon tip (see sup FigureS1) for two sample temperatures.
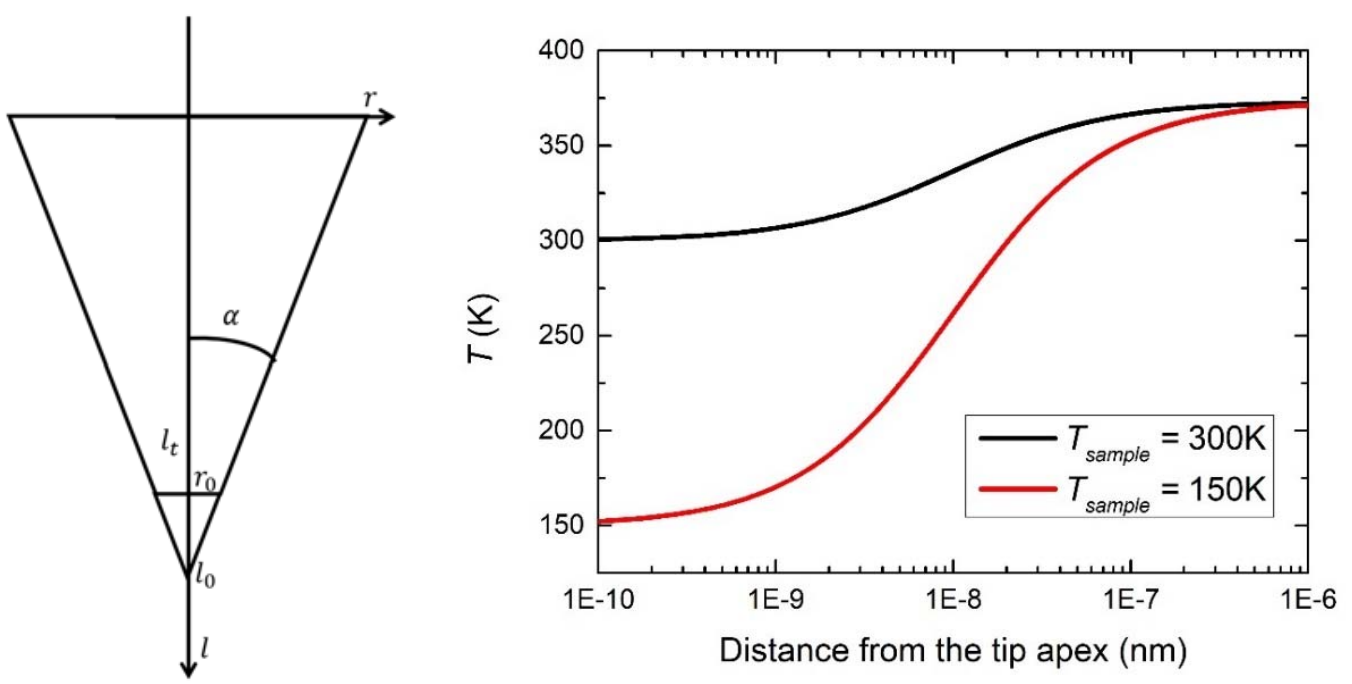

Figure S1. (a) Schematic representation of the tip. (b) Temperature distribution from the tip apex of the conical tip for 2 different sample temperatures.

\subsection{Thermal resistance of the DSi tip at variable sample temperatures}

We calculate the thermal resistance of the tip $\left(R_{\text {tip }}\right)$ with the model derived by: ${ }^{[1]}$

$$
R_{\text {tip }}=\frac{3}{8} \frac{1}{\tan \frac{\theta}{2}} R_{S}
$$

where $R_{S}=\frac{1}{k} \frac{4 \lambda}{3 \pi} \frac{1}{d_{0} / 2}$. This model describes the tip as a cone of half-angle $\theta$ and apex diameter $d_{0}=60 \mathrm{~nm}$. We used temperature dependent values of silicon phonon mean free path. 

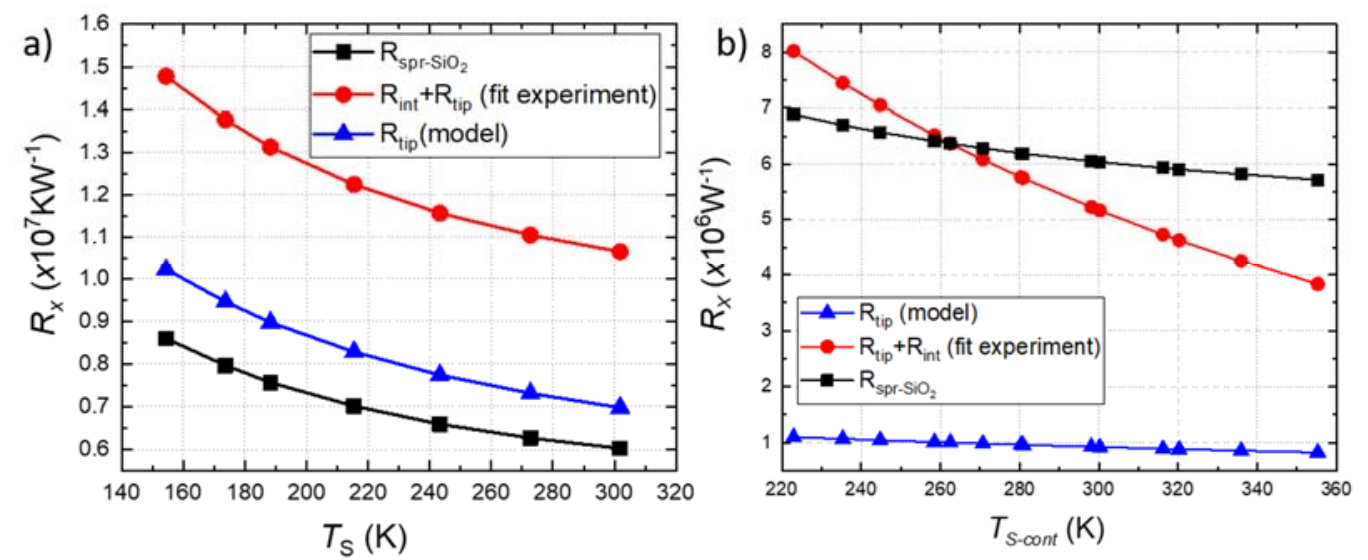

Figure S2. (a,b) Spreading resistance of $\mathrm{SiO}_{2}$ calculated by $R_{s p r-S i O 2}=1 / 2 k d_{0}$ (black). $R_{\text {tip }}+R_{\text {int }}$ obtained by fitting the experimental data to $R_{x \mathrm{SiO} 2}(T)=R_{\text {tip }}+R_{\text {int }}+R_{\text {spr-SiO2 }}$ (red). Thermal resistance of the tip as obtained from a ballistic thermal resistance by the model derived by ${ }^{[1]}$. All the above were calculated for the macroscopic temperature (a) measured during the experiment and the tip-sample contact temperature (b).

\subsection{Thermal radiation estimation}

An upper bound of the thermal radiation between the sample and the probe can be estimated by the Stefan-Boltzmann low of black body radiation given by $P=\sigma A T^{4}$, where $\sigma$ is the Stefan-Boltzmann constant, $A$ is the radiating area and $T$ the temperature. By considering the surface of the probe $\left(\sim 1000 \mu \mathrm{m}^{2}\right)$ and the maximum excess temperature $(\sim 450 \mathrm{~K})$ of the probe used in this experiment, the radiative power is found to be in the order of $10^{-15} \mathrm{~W}$. On the other hand the power of the probe heater is of the order of $10^{-3} \mathrm{~W}$ and the power injected to the sample by the end of the tip is $1 \%$ of the heater power (see section SI1). Thus thermal radiation will be approximate 9 orders of magnitude lower that the heat flux from the tip apex to sample and can be neglected. 


\section{Sample Temperature control}

During the whole experiment, the sample and the microscope temperatures $T_{S}, T_{M}$ are monitored by platinum resistance temperature detectors. The sample holder has integrated resistive heaters and is connected with a copper braid to a Liquid Nitrogen Dewar. Thus the temperature of the sample is determined by the thermal resistance of the copper braid which is connected to the liquid Nitrogen heat sink and the power dissipated by the heater. Once changing the power to the heater the system needs some time to reach equilibrium and stabilize the sample temperature.

\section{Thermal imaging speed and thermal drift}

The time per pixel of a thermal image should be larger than the thermal time constant of graphene and the response time of the probe. The characteristic times of the heat flow between the hot tip and the graphene sample is given by the thermal time constant of graphene sheet which is in the order of $0.1 \mathrm{nS}^{[2]}$ with the the response time of the probe of $\left.1 \mathrm{~ms} .^{[3]}\right]$. The time per pixel of the thermal images acquired in this study was 8-10ms making static heat transfer equations fully appropriate.”

The sample temperature is possible to drift during thermal image recording and add uncertainties to our measurements. To avoid that we record approach- and retract-curves of the SThM signal at each sample temperature immediately after every image and we compare them with ones recorded prior the image and only if they are the same we use the data. 


\section{Error analysis}

To extract the mean thermal resistance of an area, we calculate the mean thermal resistance value of the area out of the thermal resistance image. Furthermore, we calculate the Root mean squared error of the area $\left.\left(\sigma=\sqrt{\frac{1}{N} \sum_{n=1}^{N}\left(R_{x}^{i}-\bar{R}_{x}\right.}\right)\right)$ which is giving the error of the thermal resistance value.

\section{Thermal resistance of $280 \mathrm{~nm} \mathrm{SiO}_{2}$ layer on $\mathrm{Si}$}

Thermal resistance of the $\mathrm{SiO}_{2}$ layer $\left(R_{\text {layer }}\right)$ was calculated assuming isotropic layer of $\mathrm{SiO}_{2}$ on Si with diffusive transport model for thermal spreading resistance of a layer on substrate ${ }^{[4]}$ and $R_{\text {bulk }}$ from $R_{s p r}=1 / 2 k_{S} d_{0}$ for bulk sample, using temperature dependant thermal conductivity values for $\mathrm{Si}^{[5]}$ and $\mathrm{SiO}_{2}{ }^{[6]}$. As the tip diameter $\left(d_{0}\right)$ increases the contribution of the $\mathrm{Si}$ on the measured resistance increases, thus the measured resistance decreases. For $d_{0}=$ $60 \mathrm{~nm}$ the $\mathrm{SiO}_{2}$ layer thermal resistance measured is $95 \%$ of the bulk thermal resistance. The ratio of $\mathrm{SiO}_{2}$ layer to $\mathrm{SiO}_{2}$ bulk thermal resistance is similar for different sample temperatures (see Figure S3). 


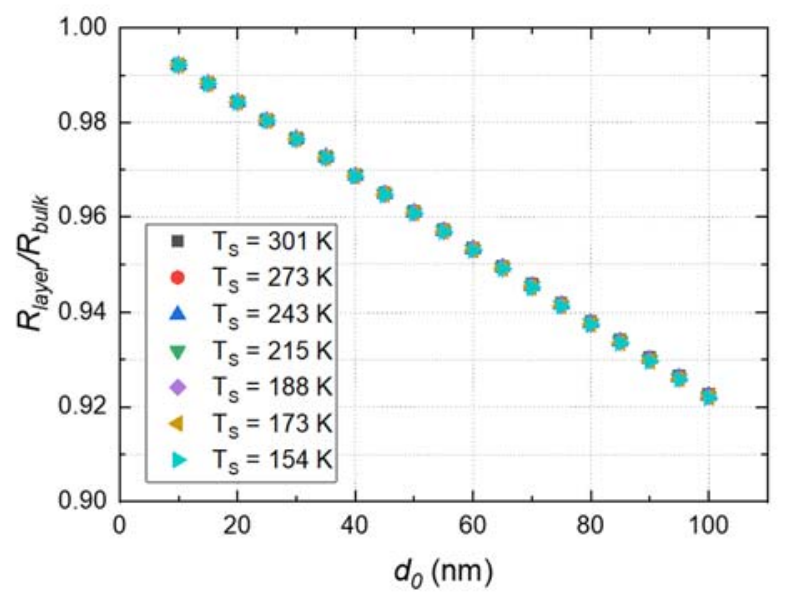

Figure S3. Ratio of Spreading resistance for $280 \mathrm{~nm} \mathrm{SiO} 2$ layer on $\mathrm{Si}$ wafer with bulk $\mathrm{SiO}_{2}$, versus tip diameter for different sample temperatures.

\section{Anisotropy of the thermal transport in the layered system}

The spreading resistance of a heat source of diameter $d_{0}$ on a layer with effective thickness $t_{\text {eff }}$ on a substrate is given by:

$R_{s p r}=\frac{1}{\pi k_{e f f} d_{0} / 2} \int_{0}^{\infty}\left[\frac{1+\operatorname{Kexp}\left(-2 \zeta^{t_{e f f}} / d_{0} / 2\right)}{1-\operatorname{Kexp}\left(-2 \zeta^{t_{e f f}} / d_{0} / 2\right)}\right] J_{1}(\zeta) \sin \zeta \frac{d \zeta}{\zeta^{2}}$,

Where $J_{1}(\zeta)$ is the Bessel function, $K=\left(1-k_{\text {sub }} / k_{\text {eff }}\right) /\left(1+k_{\text {sub }} / k_{\text {eff }}\right)$ with $k_{\text {sub }}$ being the thermal conductivity of the substrate and $k_{e f f}$ the effective thermal conductivity of the 2D material of effective thickness $t_{\text {eff }}=n t+r_{\text {int }} k_{\text {eff }}$ with $t$ being the thickness of the 2D material, $n$ the number of layers, and $r_{i n t}$ the thermal interface resistivity between the substrate and the 2D material.

An orthotropic system with directional-dependent thermal conductivities can be transformed to effective isotropic thermal conductivitiy by setting: $k_{e f f}=\sqrt{k_{c}(n) \times k_{i}(n)}$ and $k_{c}, k_{i}$ being the cross-plane and in-plane thermal conductivity of the $2 \mathrm{D}$ material respectively, $t_{e f f}=$ $n t \sqrt{k_{i}(n) / k_{c}(n)}+r_{\text {int }} k_{\text {eff }}$ with $t$ being the thickness of the 2D material and $r_{\text {int }}$ the thermal 
interface resistivity between the substrate and the $2 \mathrm{D}$ material ${ }^{[7]}$. For a single layer of a $2 \mathrm{D}$ material $k_{c}$ is not defined and therefore we assume isotropic layer of $k_{e f f}=k_{c}=k_{i}$.

\section{Lateral Resolution of the thermal images in SThM}

We estimate the thermal lateral resolution from thermal resistance profiles with two signal levels corresponding to Graphene and Graphene/MoS2. As a resolution criterion, we use the $10-90 \%$ of the lateral distance of this change. We find lateral resolution varying from $23-$ $30 \mathrm{~nm}$. Thus the tip radius is between 46 and $60 \mathrm{~nm}$ (see Figure S4).
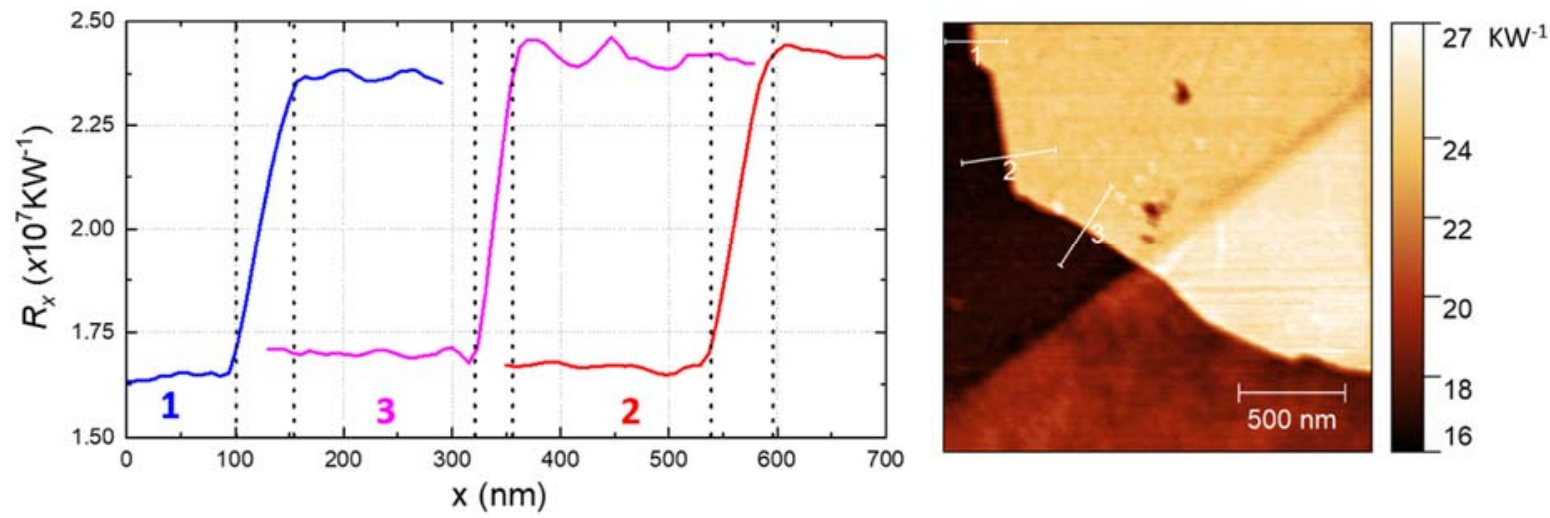

Figure S4. Thermal resistance profiles (left) obtained from the thermal image (right). With dotted lines is the $10-90 \%(55,60,46 \mathrm{~nm}$ for profile 1,2 and 3 respectively $)$ of the lateral distance where thermal resistance changes between Graphene and Graphene/MoS 2 signal level. 


\section{Thermal Resistance Measurements on Si and thermal transport in the DSi}

We use a standard Si wafer and measure thermal contact resistance $\left(R_{X}\right)$ at temperatures varying from $150-300 \mathrm{~K}$ (see Figure S5a). $R_{X}$ of Si shows a sharp increase with temperature up to $180 \mathrm{~K}$ followed by a shoulder up to $220 \mathrm{~K}$ and then decreases up to room temperature (RT). In contrast to $\mathrm{SiO}_{2}$ (see main text), the measurements with 2 different probe temperatures ( $T_{h}=350 \mathrm{~K}$ and $T_{h}=450 \mathrm{~K}$ for red and blue curve respectively) coincide, indicating that the hot tip doesn't locally heat the sample as for $\mathrm{SiO}_{2}$.
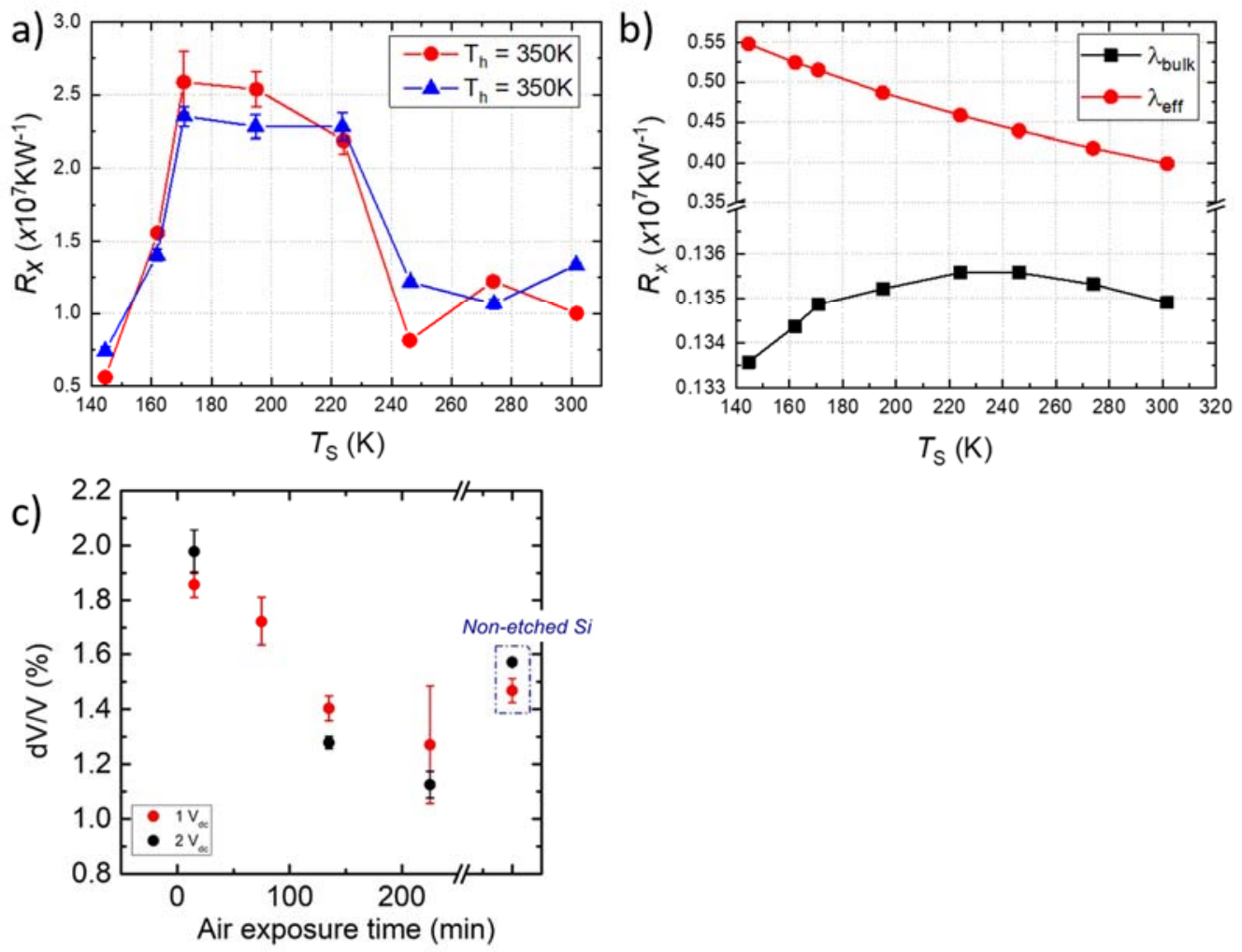

Figure S5. (a) $R_{X}$ of DSi tip in contact with $\mathrm{Si}$ at various sample temperatures with the tip heater temperature at $350 \mathrm{~K}$ (red) and $450 \mathrm{~K}$ (blue). (b) Theoretical $R_{X}$ calculated from Equation S11 for $\lambda_{\text {bulk }}$ (red) and $\lambda_{\text {eff }}$ (black). (c) SThM response in high vacuum on etched Si with time exposed to air which is related with increased native oxide thickness. 
By using the thermal contact resistance relation $R_{X S i}(T)=R_{\text {tip }}+R_{\text {int }}+1 / 2 k_{S i}(T) d_{0}$ (see main text), with temperature dependent thermal conductivity values ${ }^{[5]}$ and using $R_{\text {tip }}+R_{\text {int }}$ as a fitting parameter we are not able to reproduce the experimental trends. Si has a high $k$ and long $\lambda$ comparable with the dimensions of the tip-sample contact and both increases while lowering the temperature between $150-300 \mathrm{~K}$. Due to the comparable size of $\lambda$ and tipsample contact ballistic heat transport phenomena should be taken in to account. To understand the origin of the observed trends we examine qualitatively the different contributions in the $R_{X}$ coming from $R_{\text {int }}, R_{\text {spr }}$ and $R_{\text {tip }}$.

We first investigate the effect of the increased $\lambda$ in the sample and the tip at low temperatures. The total thermal resistance of the silicon tip - Si surface contact, at perfect contact, in the diffusion-ballistic regime can be described with a good approximation by ${ }^{[8]}$.

$R_{x c}=\frac{1}{2 k^{d_{0} / 2}}\left(1+\frac{8}{3 \pi} K_{n}\right)$,

Where $K_{n}=\frac{\lambda}{d_{0} / 2}$ is the Knudsen number, $d_{0}$ is the diameter of the contact area, $k$ the thermal conductivity of the material. We apply this model for Si-Si contact with diameter of $d_{0}=$ $60 \mathrm{~nm}$ and for temperature dependent values of $\lambda^{[9]}$ and $k^{[5]}$ ) for bulk silicon. We find that $R_{X}$ decreases with increased temperature due to the decrease of the $\lambda$ (see red curve Figure S5 b). This trend describes well our experimental results for the temperature range of $200-300 \mathrm{~K}$ but not for lower temperatures.

A second factor which could affect the $\lambda$ and therefore $R_{X}$, is the geometry of the tip-sample contact and more specifically the geometry of the DSi tip. Due to the comparable size of $\lambda$ and tip dimensions, the impinging phonons from inside the tip to the apex will have a limited $\lambda$ due to the scattering at the boundaries, and $\lambda$ is not expected to change significantly with the temperature as in the case of bulk Si. In addition, the temperature at a distance of the order of $100-200 \mathrm{~nm}$ from the tip apex in to the tip will not vary significantly from the probe heater 
temperature since more than $90 \%$ of the temperature drops in distance on the order of three times the apex diameter ${ }^{[1]}$.

It has been shown that for the calculation of the in plane $k$ of silicon thin films ${ }^{[10]}$ an effective mean free path, $\lambda_{e f f}$, should be used and described by:

$\lambda_{\text {eff }}=\lambda_{\text {bulk }}\left[1-\frac{3(1-p)}{2 \delta} \int_{1}^{\infty}\left(\frac{1}{t^{3}}-\frac{1}{t^{5}}\right) \times \frac{1-\exp (-\delta t)}{1-p \exp (-\delta t)} d t\right]$,

where $\delta=(4 / 3) l / \lambda_{\text {bulk }}$ with $l$ the thickness of the Si film, and the specularity parameter $p$, $\lambda_{\text {bulk }}$ the $\lambda$ for bulk silicon. Even though the geometrical characteristics of the tip should be taken into account, calculating $\lambda_{e f f}$ for the in-plane direction of a silicon thin film is a good approximation for a lower bound estimation. We use temperature dependent $\lambda_{\text {bulk }}$ values for the temperature of the Si tip 200nm away from the apex using as $l=d_{0}$ and $p=0$ for a totally diffuse boundary condition. We used $k$ values of bulk Si at the temperature $100 \mathrm{~nm}$ from the tip apex which will be the mean $k$ near the end of Si tip (see section 2). Then by substituting $\lambda_{e f f}$ in Equation S11 we get a more similar to the experimental trend but at lower absolute values (see black curve in Figure S5b). This discrepancy could be attributed to several factors not taken into account, such as the native $\mathrm{SiO}_{2}$ layer on the $\mathrm{Si}$ sample and the $\mathrm{Si}$ tip and the interface thermal resistance between the tip and the sample. The $\mathrm{SiO}_{2}$ layer could play a simple interface role but also could act as an extra filter on the phonon propagation, increasing or reducing the phonon modes associated with a given mean free path. Our experiment on a silicon sample etched by hydrofluoric acid, showed a significant decrease (30-40\%) of the SThM thermal response for increasing oxide thickness (see Figure S5c). The experiment was performed as follows: etched wafer was transferred in the High Vacuum SThM system and the SThM response was measured. After we repeated the following procedure for 3 times: After breaking the vacuum and exposing the sample to ambient air for a certain period of time, therefore increasing native oxide thickness, we pump the system to High Vacuum and measure the SThM response. 


\section{Graphene/MoS $\mathbf{M}_{2}$ Heterostructure}

\subsection{Fabrication}

The Graphene- $\mathrm{MoS}_{2}$ sample was fabricated by mechanical cleavage from bulk graphite and $\mathrm{MoS}_{2}$ (SPI supplies). A chip of bulk material is placed on an adhesive tape, which is used to exfoliate the material by peeling it off several times. Then the adhesive tape is brought into contact with a $\mathrm{SiO}_{2}(280 \mathrm{~nm})$ substrate thermally grown on highly doped $\mathrm{Si}$ and peeled-off slowly, leaving several flakes of different thicknesses randomly distributed on the substrate. The atomically-thin flakes are identified by optical microscopy and a pick-and-place method is used to pick-up the $\mathrm{MoS}_{2}$ monolayer, by means of a polypropylene carbonate (PPC) polydimethylsiloxane (PDMS) stamp, and transferred on top of the graphene flake afterwards with a homemade transfer setup. The PPC residues remaining on the heterostructure surface are then removed by introducing the sample in a chloroform bath.

Graphene and $\mathrm{MoS}_{2}$ are separately exfoliated on $\mathrm{SiO}_{2}(280 \mathrm{~nm})$ substrates thermally grown on highly doped Si. The monolayer flakes are identified by optical microscopy. Figure S6a shows an optical microscopy picture of a monolayer graphene flake (black dashed line) coupled to a thicker flake. Figure S6b shows an optical microscopy image of the isolated monolayer $\mathrm{MoS}_{2}$ flake (blue dashed line). The monolayer $\mathrm{MoS}_{2}$ flake is then picked-up by means of a PPC stamp and transferred on the graphene flake. An optical microscopy image of the heterostructure is shown in Figure S6c.
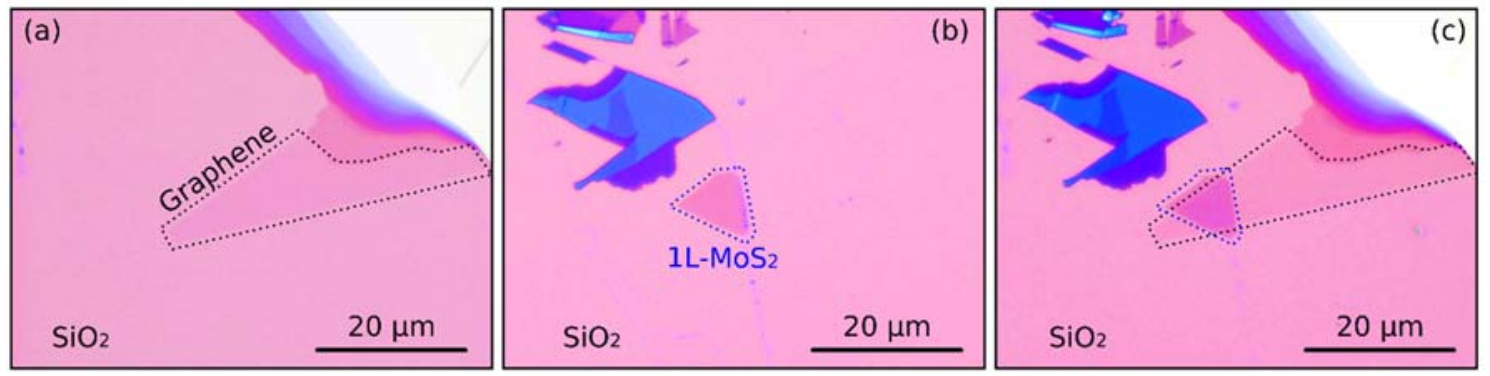
Figure S.6 Exfoliation and transfer of graphene and $\mathbf{M o S}_{2}$. (a) Optical microscopy picture of the as-exfoliated monolayer graphene on a $\mathrm{SiO}_{2}$ substrate (black dashed line). The monolayer is coupled to a thicker flake. (b) Optical microscopy picture of the as-exfoliated monolayer $\mathrm{MoS}_{2}$ (blue dashed line) on a $\mathrm{SiO}_{2}$ substrate. (c) Optical microscopy picture of the van der Waals heterostructure resulting from transferring the monolayer $\mathrm{MoS}_{2}$ (blue dashed line) on the monolayer graphene (black dashed line).

\subsection{AFM Topography}

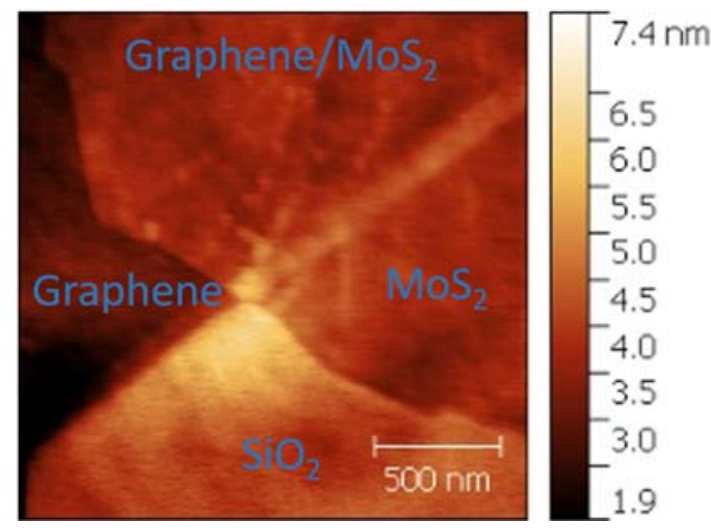

Figure S7. Topography image acquired at $220 \mathrm{~K}$ with the different areas of interest.

The thicknesses of $\mathrm{MoS}_{2}$, graphene and graphene/MoS2 heterostructure were found to be 0.7, 0.2 and $0.9 \mathrm{~nm}$ respectively. The $\mathrm{SiO}_{2}$ at the topography image appears to be higher than the Graphene/MoS 2 heterostructure. This might be a result of different friction between the two areas which causes a different torsion of the cantilever and therefore a cross-talk between the readout AFM photodetector channels ${ }^{[11]}$. A second possible reason is some a polypropylene carbonate (PPC) residues remaining on the surface as a result of the exfoliation process. Such residues will act as an extra boundary resistance on the thermal contact resistance measured on the $\mathrm{SiO}_{2}$. We can estimate the extra boundary resistance using Equation3 of the main text and considering a PPC isotropic layer of $2 \mathrm{~nm}$ with $k=0.16 \mathrm{~W} \cdot \mathrm{m}^{-1} \mathrm{~K}^{-1}{ }^{[12]}$ on $\mathrm{SiO}_{2}$ substrate. We 
find $R_{s p-P P C}=4.9 \times 10^{6} \mathrm{KW}^{-1}$, which is in the order of the spreading thermal resistance of $300 \mathrm{~nm} \mathrm{SiO}_{2}$ on $\mathrm{Si}$.

\subsection{Nanomechanical properties and morphology}

The nanomechanical study has been performed by Ultrasonic Force Microscopy (UFM), SPM method used to characterize stacked 2D materials and substructures buried under the layers. The UFM is a qualitative method in which the different contrast of the image shows diverse nanomechanical properties. Stiffer materials appear represented by brighter colours in the images ${ }^{[13]}$. The UFM image (see Figure S8) shows different contrast between the silicon substrate and the heterostructure. We observe the following trend from darker to brighter regions $\mathrm{MoS}_{2}$, Graphene, Graphene/MoS 2 heterostructure and $\mathrm{SiO}_{2}$. The fact that $\mathrm{MoS}_{2}$ on top of graphene appears brighter than $\mathrm{MoS}_{2}$ and Graphene area indicate that $\mathrm{MoS}_{2}$ is better attached to graphene than on $\mathrm{SiO}_{2}$.
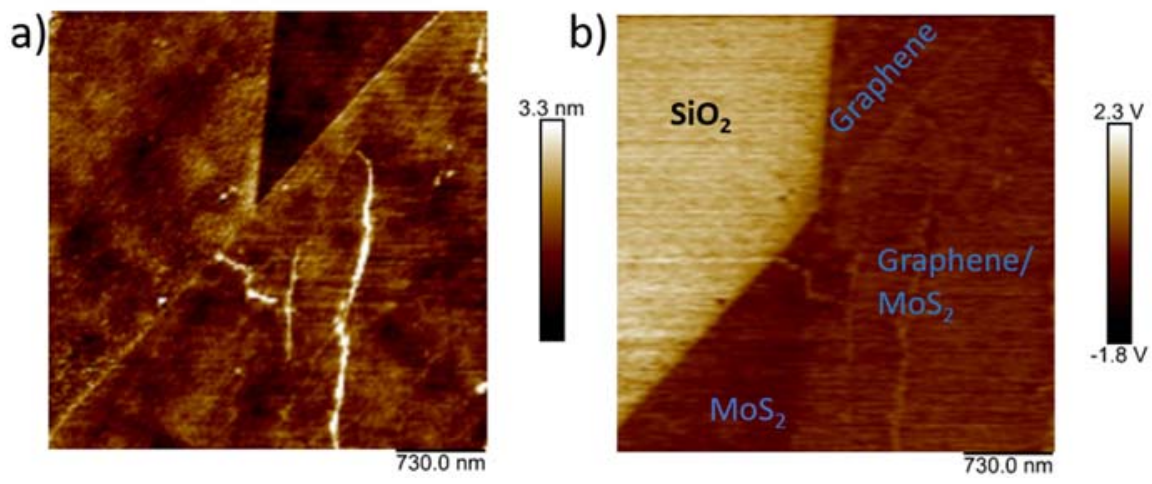

Figure S8. (a,b) Topography (a) and UFM (b) images acquired simultaneously. The different areas are shown on the UFM image. 
11. Sample local heating by the tip

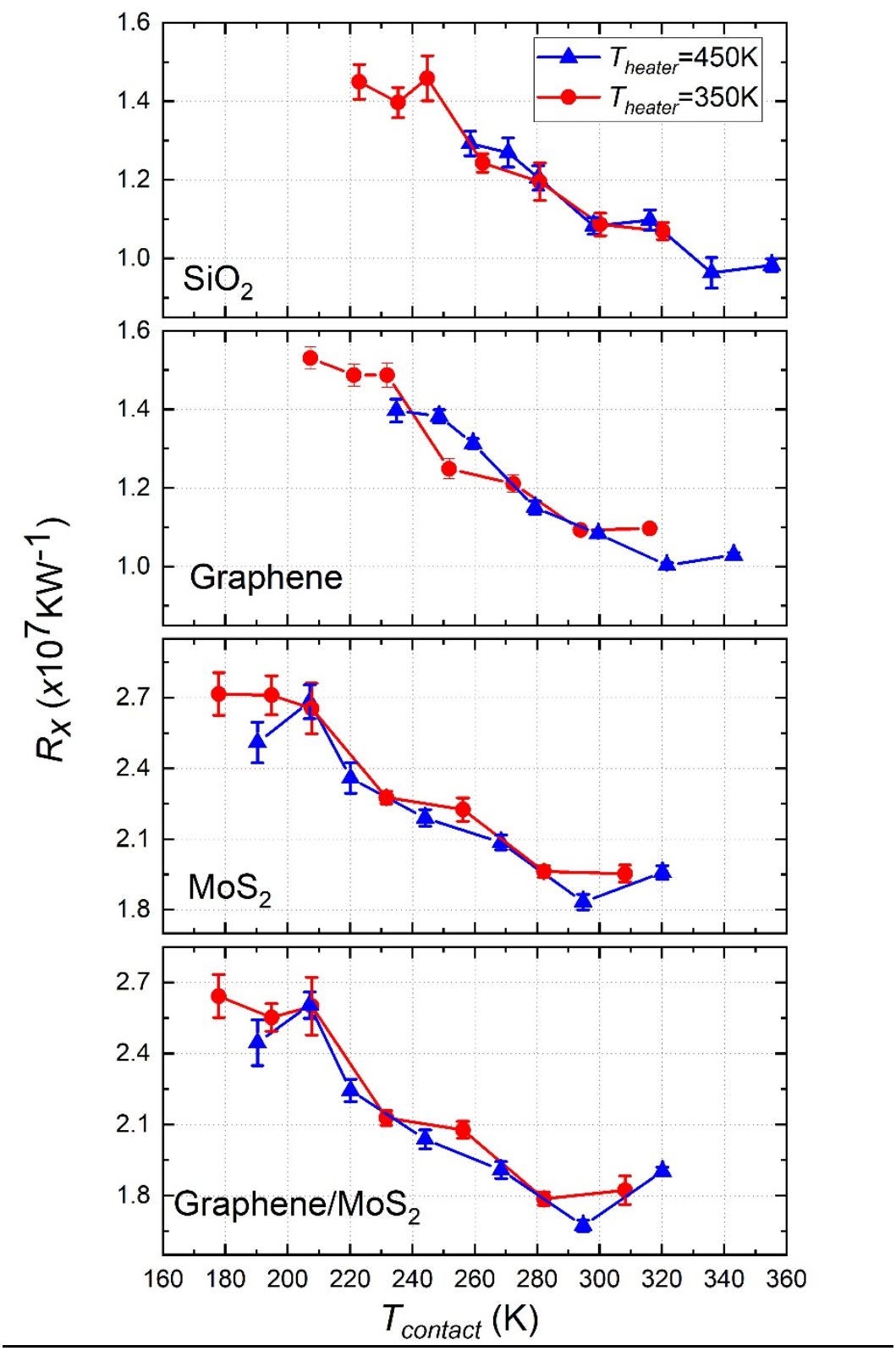

Figure S9. Thermal Resistance measured with 2 different heater temperatures versus tipsample contact temperature as obtained by the correction (see main text) for $\mathrm{SiO}_{2}$ on $\mathrm{Si}$, Graphene on $\mathrm{SiO}_{2}, \mathrm{MoS}_{2}$ on $\mathrm{SiO}_{2}$ and Graphene- $\mathrm{MoS}_{2}$ heterostructure on $\mathrm{SiO}_{2}$.

\section{SThM imaging of detached Graphene Areas}

Detached areas are formed at mechanically exfoliated graphene on different substrates in the form of ripples or bubbles ${ }^{[14]}$. These detached areas are of fundamental interest due to the 
different physical properties presenting in comparison to supported or suspended graphene sheets. The detached areas appear to be less or more thermally resistive than the attached graphene areas. Figure $\mathrm{S} 10$ a shows a $R_{X}$ image of for few layers graphene on $\mathrm{SiO}_{2}$ acquired at sample temperature, $T_{\text {sample }}=210 \mathrm{~K}$. Attached and detached areas have different thermal contrast. Topography and $R_{X}$ profiles of some detached areas (see Figure $\mathrm{S} 10 \mathrm{~b}$ ) show that the perimeter resistance is not associated with any topographical artefacts.

a)

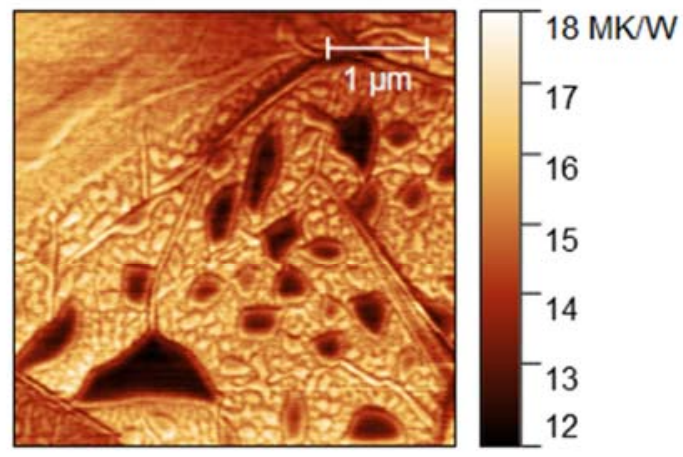

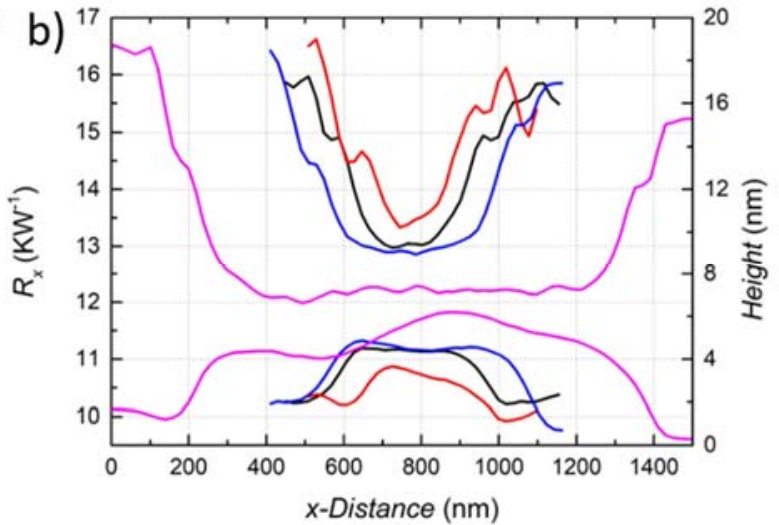

Figure S10. a) Thermal resistance image of for few layers graphene on $\mathrm{SiO}_{2}$ acquired at $\left.T_{\text {sample }}=220 \mathrm{~K} . \mathrm{b}\right)$ Topography and corresponding $R_{X}$ profiles of some detached areas.

In Figure S1 1a-d we show thermal resistance and topography images of bilayer graphene on $\mathrm{SiO}_{2}$ acquired simultaneously for the same area at 150 and $300 \mathrm{~K}$. We consider the detached areas which form bubbles as graphene disks with diameter $D$. In Figure $\mathrm{S} 12 \mathrm{a}$, b we plot the $R_{X}$ at $150 \mathrm{~K}$ and $300 \mathrm{~K}$ of the centre of each detached area of the bilayer graphene as function of its radius $(D / 2)$ as measured from the topographic images. $R_{X}$ at $150 \mathrm{~K}$ decreases with radius and stabilizes for areas larger than $50 \times 10^{3} \mathrm{~nm}$ while for $300 \mathrm{~K}$ this trend is barely visible. 
a)

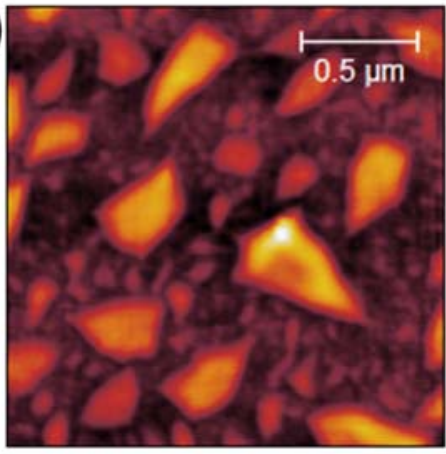

c)

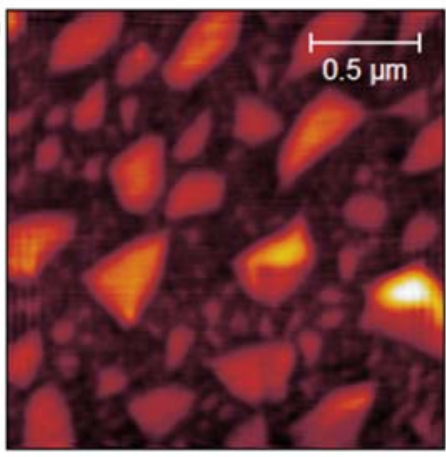

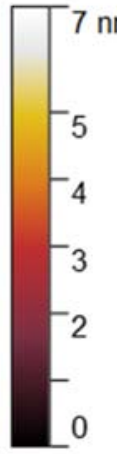
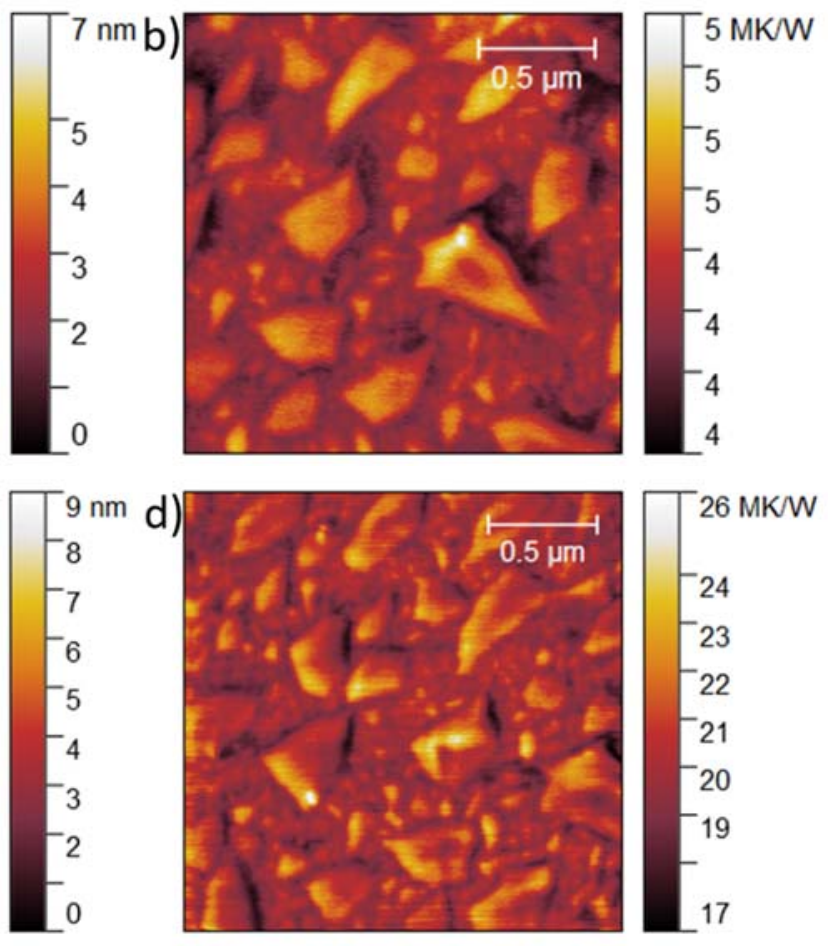

Figure S 11. (a-d) Topography $(\mathrm{a}, \mathrm{c})$ and thermal contact resitance $\left(R_{X}\right)(\mathrm{b}, \mathrm{d})$ images acquired simultaneously at $150 K(\mathrm{a}, \mathrm{b})$ and $300 K(\mathrm{c}, \mathrm{d})$ of bilayer graphene on $\mathrm{SiO}_{2}$.
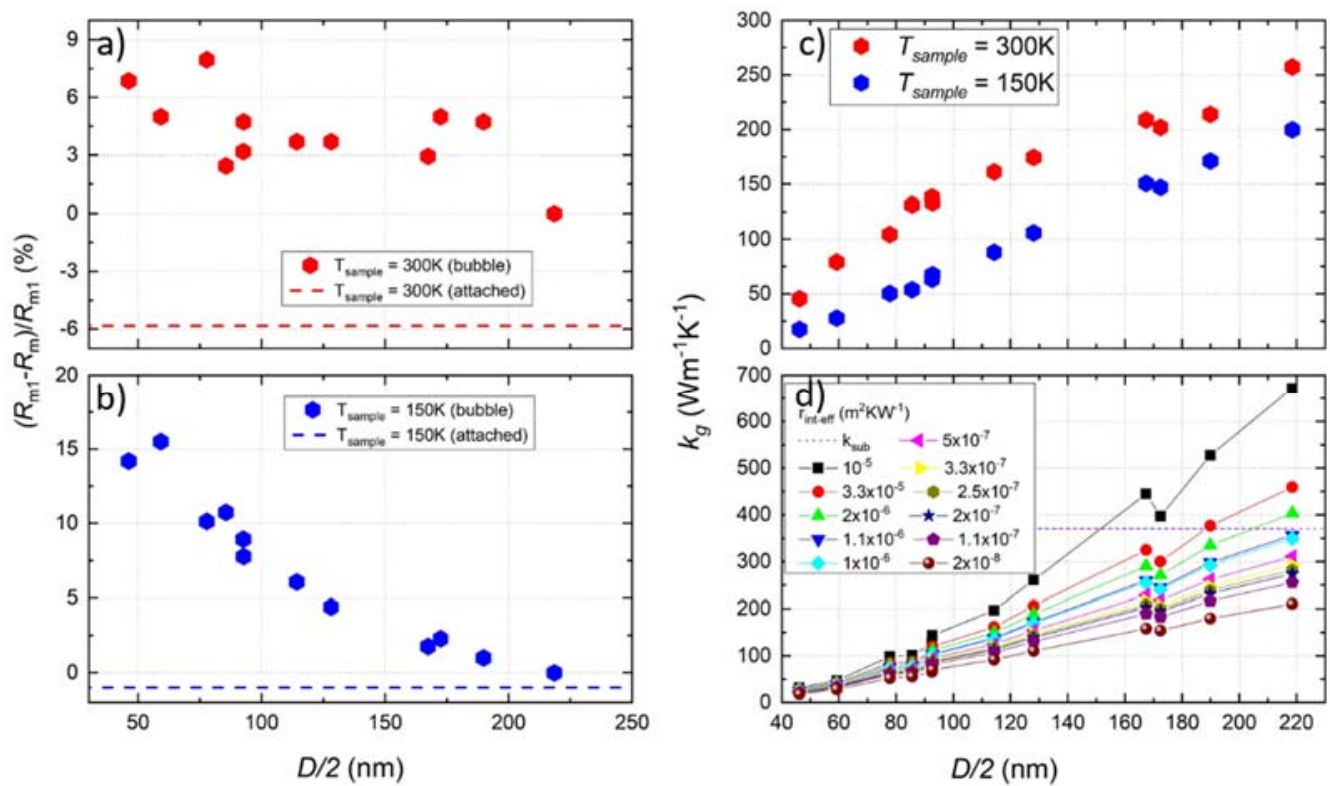

Figure S12. $(\mathrm{a}, \mathrm{b}) R_{x}$ of the center of each bubble with respect to the radius of the bubble at $300 \mathrm{~K}$ (a) and $150 \mathrm{~K}$ (b). (c) Calculated $k_{g}$ of detached areas in form of bubbles vs radius of the area at $150 \mathrm{~K}$ (blue) and $300 \mathrm{~K}$ (red). (d) Calculated $k_{g}$ for the detached areas at $150 \mathrm{~K}$ using different values of effective thermal resistivity. 
We model the detached graphene areas as suspended graphene disks with diameter $D$ and use the model proposed by ${ }^{[15]}$. They modelled the $R_{s p r}$ from the center of a graphene disk to the substrate as the sum of the thermal spreading resistance from the center of the disk to the edge $\left(R_{b u b}\right)$ and that from the edge to the substrate $\left(R_{e s}\right)$ :

$$
R_{b-t o t}=R_{b u b}+R_{e s}=\frac{\ln (D / 2)-\ln \left(d_{0} / 2\right)}{2 \pi t k_{g}}+\frac{1}{2 \pi(D / 2)} \sqrt{\frac{r_{i n t}}{t k_{S}}} \frac{K_{0}\left(z_{D / 2}\right)}{K_{1}\left(z_{D / 2}\right)}
$$

where $t$ is the thickness, $r_{\text {int }}$ is the interface thermal resistivity between graphene and $\mathrm{SiO}_{2}$, $k_{s}$ and $k_{g}$ is the thermal conductivity of supported and suspended graphene respectively and $z_{D / 2}=(D / 2) \sqrt{1 / r_{i n t} k_{s} t}$

We should comment that this model does not describe precisely the detached areas in the form of bubbles of our sample. First they have random shapes which we approximate as disks and secondly between the detached graphene and the $\mathrm{SiO}_{2}$ substrate some gases might present which could affect the thermal transport. Nevertheless, it gives a better qualitative understanding of our experimental results.

Combining Equation 2 of the main text and EquationS13, we can calculate $k_{g}$ for the different detached areas of graphene as a function of $D / 2$ (see Figure S12c). Considering that the interface resistance of the $\mathrm{SiO}_{2}$ covered DSi tip and the Graphene $\left(R_{\text {int }(S G)}\right)$ is the same in the attached and detached areas we can extract $R_{\text {int-tot }(S G)}=R_{\text {tip }}+R_{\text {int }(S G)}$ by combining Equation 2 and 3 of the main text for the tip being on top of the attached area and therefore, $R_{\text {int-tot }(S G)}=R_{x}-R_{s p r}$. We use literature values for $k_{g}{ }^{[16]}$ and $k_{c}(2)^{[17]}$ for 2-layers graphene. We find that $k_{g}$ increases linearly with $D / 2$ at both temperatures. $k_{g}$ is higher for higher temperatures in good agreement with previous studies for graphene ${ }^{[18]}$. The thermal conductivity of both attached and detached areas increases from $150 \mathrm{~K}$ to $300 \mathrm{~K}$. 
Interestingly, $k_{g}$ of different detached areas are smaller than $k_{s}$ in both temperatures. This contradicts with previous results ${ }^{[15]}$. Possible reasons for that is the perimeter thermal resistance, $R_{p e r}$, of each detached area. $R_{\text {per }}$ is evident sometimes in the thermal resistance image (see Figure S10a), where the perimeter of the detached area appears as a brighter ring, indicating a more resistive region. The perimeter resistance of the bubble of interest as well as of the surrounding bubbles will act as additional interface resistances for the $R_{\text {sup }}$. Therefore, an effective boundary thermal resistivity $r_{\text {int-eff }}$, which includes the contribution of the thermal boundary resistivity of the perimeter of the bubble $\left(r_{i n t-p}\right)$ and of the surrounding detached areas $\left(r_{i n t-s}\right)$ as well as for the attached area $\left(r_{i n t}\right)$ in the form $r_{\text {int-eff }}=r_{i n t-p}+$ $r_{i n t-s p}+r_{i n t}$ should be used. By using arbitrary $r_{i n t-e f f}$ up to 2 orders of magnitude higher than values from ref. ${ }^{[19]}$ we get $k_{g}>k_{s}$ for the bigger bubbles and for high $r_{\text {int-eff }}$ (see Figure S12d).

Further we comment on the different thermal contrast between attached and detached areas of graphene we get depending on the tip size. For larger radius tip, thus larger tip-sample contact area, the bubbles tend to have higher $R_{X}$ than the supported graphene and for smaller radius lower. The fact that we see this behavior even for the large bubbles (much larger than the contact area) make us exclude the possibility of contact area image artefacts. When the tip is scanning through detached and attached areas the $R_{X}$ is given by Equation 2 of main text. $R_{\text {tip }}$ and $R_{\text {int }}$ are not expected to change from the attached to detached area of scanning. Thus, the contrast depends on the relative spreading resistance of the attached $\left(R_{e s}\right)$ and detached $\left(R_{b u b}\right)$ areas given by eq.S11. Indeed, calculating the $R_{s p r}$ and $R_{s p r-d e t}$ for different tip radius and different $r_{\text {int-eff }}$ the relative change of $R_{b u b}$ to $R_{e s}\left(\left(R_{e s}-R_{b u b}\right) / R_{e s}\right)$ is changing sign from positive to negative while increasing the tip radius (see Figure S13). 


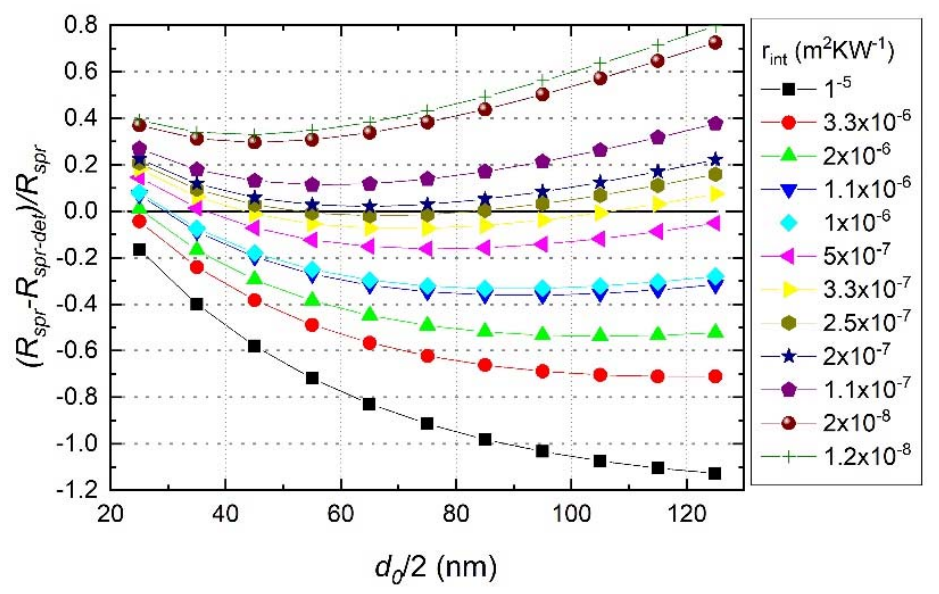

Figure S13. Relative change of attached and detached areas spreading resistance, $\left.\left(R_{e s}-R_{b u b}\right) / R_{e s}\right)$, versus tip radius for calculated for different interface resistances.

\section{REFERENCES.}

[1] B. Gotsmann, M. A. Lantz, A. Knoll, U. Dürig, in Nanotechnology, DOI: 10.1002/9783527628155. nanotech066, Wiley-VCH Verlag GmbH \& Co. KGaA 2010.

[2] E. Pop, V. Varshney, A. K. Roy, MRS Bull. 2012, 37, 1273.

[3] P. Tovee, M. Pumarol, D. Zeze, K. Kjoller, O. Kolosov, J. Appl. Phys. 2012, 112, 114317.

[4] M. M. Yovanovich, J. R. Culham, P. Teertstra, IEEE Transactions on Components, Packaging, and Manufacturing Technology: Part A 1998, 21, 168.

[5] C. J. Glassbrenner, G. A. Slack, Phys. Rev. 1964, 134, A1058.

[6] M. Anis-ur-Rehman, A. Maqsood, Int. J. Thermophys. 2003, 24, 867.

[7] a) Y. S. Muzychka, M. M. Yovanovich, J. R. Culham, J. Thermophys Heat Transfer 2004, 18, 45;

b) F. Menges, H. Riel, A. Stemmer, C. Dimitrakopoulos, B. Gotsmann, Phys. Rev. Lett. 2013, 111, 205901.

[8] R. Prasher, Nano Lett. 2005, 5, 2155.

[9] R. S. Prasher, P. E. Phelan, J. Appl. Phys. 2006, 100, 063538.

[10] C. Jeong, S. Datta, M. Lundstrom, J. Appl. Phys. 2012, 111, 093708.

[11] A. Hoffmann, T. Jungk, E. Soergel, Rev. Sci. Instrum. 2007, 78, 016101.

[12] M. Tuliszka, F. Jaroszyk, M. Portalski, Int. J. Thermophys. 1991, 12, 791.

[13] B. J. Robinson, C. E. Giusca, Y. T. Gonzalez, N. D. Kay, O. Kazakova, O. V. Kolosov, 2D Materials 2015, 2.

[14] a) K. Yue, W. Gao, R. Huang, K. M. Liechti, J. Appl. Phys. 2012, 112; b) T. Georgiou, L. Britnell, P. Blake, R. V. Gorbachev, A. Gholinia, A. K. Geim, C. Casiraghi, K. S. Novoselov, Appl. Phys. Lett. 2011, 99.

[15] G. Hwang, O. Kwon, Nanoscale 2016, 8, 5280.

[16] M. M. Sadeghi, I. Jo, L. Shi, Proc Natl Acad Sci U S A 2013, 110, 16321.

[17] A. Alofi, G. P. Srivastava, Appl. Phys. Lett. 2014, 104. 
[18] X. Xu, L. F. Pereira, Y. Wang, J. Wu, K. Zhang, X. Zhao, S. Bae, C. Tinh Bui, R. Xie, J. T. Thong, B. H. Hong, K. P. Loh, D. Donadio, B. Li, B. Ozyilmaz, Nat Commun 2014, 5, 3689.

[19] P. Yasaei, C. J. Foss, K. Karis, A. Behranginia, A. I. El-Ghandour, A. Fathizadeh, J. Olivares, A. K. Majee, C. D. Foster, F. Khalili-Araghi, Z. Aksamija, A. Salehi-Khojin, Advanced Materials Interfaces 2017, 4, 1700334. 